\title{
XMM-Newton Spectroscopy of the Cluster of Galaxies 2A 0335+096
}

\author{
N. Werner ${ }^{1}$, J. de Plaa ${ }^{1,2}$, J.S. Kaastra ${ }^{1}$, Jacco Vink ${ }^{1,2}$, J. A. M. Bleeker ${ }^{1,2}$, T. Tamura ${ }^{3}$, J. R. Peterson ${ }^{4}$, and F. \\ Verbunt $^{2}$
}

1 SRON Netherlands Institute for Space Research, Sorbonnelaan 2, NL - 3584 CA Utrecht, the Netherlands

2 Astronomical Institute, Utrecht University, P.O. Box 80000, NL - 3508 TA Utrecht, the Netherlands

3 Institute of Space and Astronautical Science, JAXA, 3-1-1 Yoshinodai, Sagamihara, Kanagawa 229-8510, Japan

4 KIPAC, Stanford University, PO Box 90450, MS 29, Stanford, CA 94039, USA

\begin{abstract}
We present here the results of a deep (130 ks) XMM-Newton observation of the cluster of galaxies 2A $0335+096$. The deep exposure allows us to study in detail its temperature structure and its elemental abundances. We fit three different thermal models and find that the multi-temperature wdem model fits our data best. We find that the abundance structure of the cluster is consistent with a scenario where the relative number of Type Ia supernovae contributing to the enrichment of the intra-cluster medium is $\sim 25 \%$, while the relative number of core collapse supernovae is $\sim 75 \%$. Comparison of the observed abundances to the supernova yields does not allow us to put any constrains on the contribution of Pop III stars to the enrichment of the ICM. Radial abundance profiles show a strong central peak of both Type Ia and core collapse supernova products. Both the temperature and iron abundance maps show an asymmetry in the direction of the elongated morphology of the surface brightness. In particular the temperature map shows a sharp change over a brightness edge on the southern side of the core, which was identified as a cold front in the Chandra data. This suggests that the cluster is in the process of a merger with a subcluster. Moreover, we find that the blobs or filaments discovered in the core of the cluster by Chandra are, contrary to the previous results, colder than the ambient gas and they appear to be in pressure equilibrium with their environment.
\end{abstract}

Key words. Galaxies: clusters: general - Galaxies: clusters: individual: 2A 0335+096 - cooling flows - intergalactic medium - Galaxies: abundances - X-rays: galaxies: clusters

\section{Introduction}

Clusters of galaxies are the largest known gravitationally bound structures in the universe. According to the standard cosmological scenario they form and grow along the filaments through merging with groups and individual galaxies. Optical and Xray studies reveal that clusters of galaxies are still forming at the present epoch.

The large effective area and superb spectral resolution of XMM-Newton together with the high spatial resolution of Chandra allow us to study clusters of galaxies with unprecedented detail. Analysis of data obtained by these satellites led to a number of important results in the recent years. In the central parts of many clusters of galaxies the gas density is high enough that the radiative cooling time of the gas is shorter than the age of the cluster. As the gas cools the pressure decreases, which causes a net inflow toward the center of the cluster. Many clusters of galaxies indeed show a temperature drop by a factor of three or more within the central $100 \mathrm{kpc}$ radius (for a review on cooling flows see Fabian 1994). However, the spectra obtained by the XMM-Newton Reflection Grating Spectrometer

Send offprint requests to: N. Werner, email n.werner@sron.nl
(RGS) show no evidence for strong cooling rates of gas below $30-50 \%$ of the maximum temperature of the ambient gas, which forces us to look for additional heating mechanisms in the cores of clusters (Peterson et al. 2001; Tamura et al. 2001b; Kaastra et al. 2001). The high resolution images from Chandra led recently to the discovery of cold fronts, associated with motion of the cluster cores and to the identification of filamentary structure in the cores of a number of clusters (Markevitch et al. 2000). Spatially resolved spectroscopy of many clusters of galaxies shows a strongly centrally peaked distribution of metal abundances (Tamura et al. 2004).

Because of their large potential wells, clusters of galaxies retain all the enriched material produced in the member galaxies. This makes them a unique environment for elementalabundance measurements and for the study of the chemical enrichment history of the universe.

In this paper we study the X-ray bright cluster 2A 0335+096 using spatially-resolved and high-resolution spectra obtained during a $130 \mathrm{ks}$ observation with the European Photon Imaging Camera (EPIC, Turner et al. 2001; Strüder et al. 2001) and the Reflection Grating Spectrometer (RGS, den Herder et al. 2001) aboard XMM- 
Newton (Jansen et al. 2001). The properties of 2A 0335+096 allow us to address here some of the above mentioned issues.

2A $0335+096$ was first detected as an X-ray source by Ariel V (Cooke et al. 1978), and was found to be associated with a medium compact Zwicky cluster (Zwicky et al. 1965; Schwartz et al. 1980). The presence of a cooling flow was first noted by Schwartz et al. (1980) in the data obtained by HEAO 1. X-ray observations with EXOSAT (Singh et al. 1986, 1988) and Einstein (White et al. 1991) confirmed the presence of the cooling flow. Observations with ROSAT (Sarazin et al. 1992; Irwin \& Sarazin 1995) show a filamentary structure in the central region of the cooling flow. Observations with ASCA (Kikuchi et al. 1999) show a hint of a centrally peaked metallicity distribution. Using data obtained by BeppoSAX, De Grandi \& Molendi (2001, 2002) analyzed the metallicity and temperature profile of the cluster and found a centrally peaked metallicity gradient. Using the same dataset, Ettori et al. (2002) estimated the total mass of the cluster within the region with overdensity of 2500 times the critical density to be $\sim 1.6 \times 10^{14} \mathrm{M}_{\odot}$, while the mass of the gas was found to be $\sim 2.0 \times 10^{13} \mathrm{M}_{\odot}$.

Recent Chandra observation shows a complex structure in the core of the cluster: a cold front south of the center, unusual X-ray morphology consisting of a number of X-ray blobs and/or filaments on scales $\geq 3 \mathrm{kpc}$, along with two prominent X-ray cavities (Mazzotta et al. 2003). Moreover, the Chandra observation shows that the cluster has a cool dense core and its radial temperature gradient varies with position angle. The radial metallicity profile has a pronounced central drop and an off-center peak (Mazzotta et al. 2003). A previous shorter observation with XMM-Newton shows an increase of the $\mathrm{Fe}$ abundance toward the center with a strong central peak Tamura et al. 2004). The central galaxy of 2A $0335+096$ is a cD galaxy with a very extended optical emission line region ( $\mathrm{H} \alpha+[\mathrm{NII}])$ to the northeast of the galaxy. Moreover, the central region of the galaxy is anomalously blue, indicating recent star formation (Romanishin \& Hintzen 1988). Edge (2001) reports a detection of $\mathrm{CO}$ emission (implying $2 \times 10^{9} \mathrm{M}_{\odot}$ of molecular gas) and IRAS $60 \mu \mathrm{m}$ continuum. These observations indicate a mass deposition rate of a cooling flow of $<5 \mathrm{M}_{\odot} \mathrm{yr}^{-1}$. A radio study of 2A $0335+096$ shows a radio source coincident with the central galaxy, which is surrounded by a mini-halo (Sarazin et al. 1995).

Throughout the paper we use $H_{0}=70 \mathrm{~km} \mathrm{~s}^{-1} \mathrm{Mpc}^{-1}, \Omega_{M}=$ $0.3, \Omega_{\Lambda}=0.7$, which imply a linear scale of $42 \mathrm{kpc} \mathrm{arcmin}^{-1}$ at the cluster redshift of $z=0.0349$. Unless specified otherwise, all errors are at the $1 \sigma$ confidence level.

\section{Observations and data reduction}

2A $0335+096$ was observed with XMM-Newton on August 4th and 5th 2003 with a total exposure of $130 \mathrm{ks}$. The EPIC MOS and pn instruments were operated in Full Frame mode using the thin filter. The exposure times of both MOS cameras and RGS were $130 \mathrm{ks}$ and the exposure time of pn was $93 \mathrm{ks}$.

\subsection{EPIC analysis}

The raw data are processed with the 6.0.0 version of the XMMNewton Science Analysis System (SAS), using the EPPROC and EMPROC tasks. We apply the standard filtering, for EPIC/MOS keeping only single, double, triple and quadruple pixel events (PATTERN $\leq 12$ ), while for EPIC/pn we make use of single and double events (PATTERN $\leq 4)$. In both cases only events with $\mathrm{FLAG}==0$ are considered. The redistribution and ancillary response files are created with the SAS tasks rmfgen and arfgen for each camera and region we analyze.

In the spectral analysis we remove all the bright X-ray point-sources with a flux higher than $4.8 \times 10^{-14} \mathrm{erg} \mathrm{s}^{-1} \mathrm{~cm}^{-2}$.

At the low energies, the EPIC cameras are currently not well calibrated below $0.3 \mathrm{keV}$. For pn we take a conservative lower limit of $0.5 \mathrm{keV}$, below which, at the time of our analysis, there are still some uncertainties in calibration. At energies higher than $10 \mathrm{keV}$ our spectra lack sufficient flux. Therefore, in most of the analyzed regions, our analysis of the MOS and pn spectra is restricted to the $0.3-10 \mathrm{keV}$ and $0.5-10 \mathrm{keV}$ range respectively. Bonamente et al. (2005) showed that the systematic uncertainties of the EPIC detectors in the energy range of 0.3 to $1 \mathrm{keV}$ are less than $10 \%$. We include systematic errors to account for the uncertainties in the calibration and background in the spectral fit. The applied systematics are adopted from Kaastra et al. (2004). After a preliminary analysis we find that the pn instrument has a small gain problem. We minimize this effect by shifting the pn energy grid by $10 \mathrm{eV}$. The spectra obtained by MOS1, MOS2 and pn are then fitted simultaneously with the same model, while their relative normalizations are left as free parameters.

To test whether our abundance measurements can be influenced by narrow band regions where the calibration of EPIC might still be problematic, we fit the spectrum of PKS 2155304, a bright BL Lac object observed with thin filter on 2004 November 23. The X-ray spectra of BL Lac objects are generally well fitted by simple absorbed power-law, or broken power-law models. We find that the fit residuals at the position of the measured spectral lines are always consistent within the $1 \sigma$ uncertainty with the best fit absorbed power-law model. Thus, we can rule out the possibility that the measured abundances are seriously influenced by narrow band calibration uncertainties.

\subsubsection{Background modeling}

Since we want to investigate the cluster properties up to at least $9^{\prime}$ from its core, we need a good estimate for the background, especially at the dim outer parts of the cluster. The source fills the entire field of view, which makes a direct measurement of the local background very difficult. The commonly used combined background event lists of Lumb et al. (2002) and Read \& Ponman (2003), can be used in areas where the surface brightness of the source is high, or when the cluster has similar background properties as the combined background fields. As shown by de Plaa et al. (2006) relatively small systematic errors in the background normalization can have a big influence on the spectral fits in the outer parts of clusters. 
Table 1. The CXB components used in the fitting of $2 \mathrm{~A} 0335+096$. The power-law index of the extragalactic component is frozen to $\gamma=1.41$. The fluxes are determined in the $0.3-10 \mathrm{keV}$ band and, apart of the Local hot bubble, corrected for Galactic absorption using a column of $N_{\mathrm{H}}=2.5 \times 10^{21} \mathrm{~cm}^{-2}$ (the local hot bubble is inside of the Galactic absorbing column).

\begin{tabular}{lll}
\hline \hline Component & $\mathrm{k} T(\mathrm{keV})$ & $\mathrm{Flux}\left(\mathrm{erg} \mathrm{s}^{-1} \mathrm{~cm}^{-2} \mathrm{deg}^{-2}\right)$ \\
\hline Local bubble & 0.082 & $3.58 \times 10^{-12}$ \\
Soft distant component & 0.068 & $6.57 \times 10^{-14}$ \\
Hard distant component & 0.127 & $6.19 \times 10^{-13}$ \\
Extra galactic power-law & & $2.16 \times 10^{-11}$ \\
\hline
\end{tabular}

The background can be divided into three main components: instrumental background, low energy particles (with energy of a few tens of keV) accelerated in the Earth's magnetosphere (so called soft-protons) and the Cosmic X-ray Background (CXB).

To minimize the effect of soft protons in our spectral analysis, we cut out time intervals where the total $10-12 \mathrm{keV}$ count rate deviates from the mean by more than $3 \sigma$. The cleaned MOS1, MOS2 and pn event files have useful exposure times of $107 \mathrm{ks}, 108 \mathrm{ks}$ and $77 \mathrm{ks}$, respectively.

To deal with the instrumental background we adopt a method developed by de Plaa et al. (2006). As a template for the instrumental background we use data from closed filter observations, which we scale to the source observation using events detected outside of the field of view (the values of the scaling factors are $1.00 \pm 0.02,1.04 \pm 0.02$ and $1.10 \pm 0.07$ for MOS1, MOS2 and pn respectively). The scaling is performed by adding or subtracting a powerlaw with a photon index of $\gamma=0.15$ for MOS and $\gamma=0.24$ for pn. For detailed description of the method see de Plaa et al. (2006).

We correct for the Cosmic X-ray Background (CXB) during the fitting. Since all the CXB photons enter the mirrors together with the source photons, we can fit the CXB simultaneously with the source spectra. In our models, we use CXB components described by Kuntz \& Snowden (2000). They distinguish 4 different components: the extragalactic power law (EPL), the local hot bubble (LHB), the soft distant component (SDC) and the hard distant component (HDC). The EPL component is made from the integrated emission of faint discrete sources, mainly from distant Active Galactic Nuclei (AGNs). According to one of the most recent determinations by De Luca \& Molendi (2004) the power law index of the EPL is $1.41 \pm 0.06$ and its $2-10 \mathrm{keV}$ flux is $2.24 \pm 0.16 \times 10^{-11}$ erg cm$~^{-2} \mathrm{~s}^{-1} \mathrm{deg}^{-2}$ (with $90 \%$ confidence). Since in our spectral analysis we cut out all the point sources with a flux higher than $4.8 \times 10^{-14} \mathrm{erg} \mathrm{s}^{-1} \mathrm{~cm}^{-2}$ we reduce the EPL flux in our extraction area. Moretti et al. (2003) have made a compilation of number counts of X-ray point sources in two energy bands $(0.5-2$ and $2-10 \mathrm{keV})$ from a large source sample and determined analytic formula for the number of point sources $N(S)$ with a flux higher then $S$. To determine, the contribution of the point sources brighter then our cut-off to the total flux of the
EPL we calculate the integral $\int_{S_{\text {cut-off }}}^{\infty}\left(\frac{d N}{d S}\right) S d S$. We find, that the point sources we cut out make up $\approx 20 \%$ of the total EPL flux. The $0.3-10 \mathrm{keV}$ flux of the EPL in our model is thus $2.16 \times 10^{-11} \mathrm{erg} \mathrm{cm}^{-2} \mathrm{~s}^{-1} \mathrm{deg}^{-2}$. The LHB is a local supernova remnant, in which our Solar System resides. It produces virtually unabsorbed emission at a temperature of $\sim 10^{6} \mathrm{~K}$. The SDC and HDC originate at a larger distance, they might be identified with the Galactic halo, Galactic corona or the Local group emission and are absorbed by almost the full Galactic column density. They have a temperature of $1-2$ million $\mathrm{K}$. We model each of the three soft background components (the LHB, SDC and the HDC) by a MEKAL model with temperatures of $0.082 \mathrm{keV}, 0.068 \mathrm{keV}, 0.127 \mathrm{keV}$ respectively (based on Kuntz \& Snowden 2000). We determine the normalizations of these soft components by fitting the spectrum extracted from an annulus, with inner and outer radii of $9^{\prime}$ and $12^{\prime}$ respectively, centered at the core of the cluster. The contribution to the total flux from cluster emission at these radii is $\sim 60 \%$, the rest of the emission comes from the CXB. To account for the cluster emission we use an additional thermal model. In our final spectral fits we fix the normalizations of the background components. The temperatures and the $0.3-10 \mathrm{keV}$ fluxes of the background components are given in Table 1$]$

\subsection{RGS analysis}

We extract the RGS spectra with SAS version 6.1.0 following the same method as described in Tamura et al. (2001a). In order to get spatial information we select the events from several rectangular areas on the CCD strip in the cross-dispersion direction. Because the cluster fills the entire field-of-view of the RGS, we need a blank field observation to extract the background spectrum. For this observation we choose a Lockman Hole observation with an effective exposure time of $100 \mathrm{ks}$. The flare subtraction is analogous to the method used with EPIC, but now we use the events from CCD 9 outside the central area with a cross-dispersion of $|x d s p|>30^{\prime \prime}$ to make the lightcurve. This method was applied to both source and background datasets.

Because the RGS gratings operate without a slit, the resulting spectrum of an extended source is the sum of all spectra in the (in our case) $5^{\prime} \times \sim 12^{\prime}$ field of view, convolved with the PSF (see Davis 2001, for a complete discussion about grating responses). Extended line-emission appears to be broadened depending on the spatial extent of the source along the dispersion direction. In order to describe the data properly, the spectral fits need to account for this effect. In practice, this is accomplished by convolving the spectral models with the surface brightness profile of the source along the dispersion direction (Tamura et al. 2004). For that purpose we derive for each extraction region the cluster intensity profile from MOS1 in the $0.8-1.4 \mathrm{keV}$ band along the dispersion direction of RGS and we convolve this MOS1 profile with the RGS response in order to produce a predicted line spread function (lsf). Because the radial profile for an ion can be different from the mean MOS1 profile, this method is not ideal. Therefore we introduce two scale parameters, namely the width and centroid of the lsf. 
These parameters are left free during spectral fitting in order to match the observed profiles of the main emission lines. The scale parameter $s$ for the width is the ratio of the observed lsf width to the nominal MOS1 based lsf width.

\section{Spectral models}

For the spectral analysis we use the SPEX package Kaastra et al. 1996). We model the Galactic absorption using the hot model of that package, which calculates the transmission of a plasma in collisional ionisation equilibrium with cosmic abundances. We mimic the transmission of a neutral plasma by putting its temperature to $0.5 \mathrm{eV}$. To find the best description of the cluster emission we fit several combinations of collisionally ionised equilibrium (CIE) plasma models (MEKAL) to the spectra: a single-temperature thermal model; a combination of two thermal models; a differential emission measure (DEM) model with a cut-off power-law distribution of emission measures versus temperature (wdem). The wdem model appears to be a good empirical approximation for the spectrum in cooling cores of clusters of galaxies (e.g. Kaastra et al. 2004; de Plaa et al. 2004). The emission measure $Y=\int n_{\mathrm{e}} n_{\mathrm{H}} d V$ (where $n_{\mathrm{e}}$ and $n_{\mathrm{H}}$ are the electron and proton densities, $V$ is the volume of the source) in the wdem model is shown in Eq. (1) adapted from Kaastra et al. (2004):

$\frac{d Y}{d T}= \begin{cases}A T^{1 / \alpha} & T_{\min }<T<T_{\max }, \\ 0 & \text { elsewhere. }\end{cases}$

The emission measure distribution has a cut-off at $T_{\min }=$ $c T_{\max }$. The cut-off $c$ is set in this study to 0.1. For $\alpha \rightarrow \infty$ we obtain a flat emission measure distribution. The emission measure weighted mean temperature $T_{\text {mean }}$ is given by:

$T_{\text {mean }}=\frac{\int_{T_{\min }}^{T_{\max }} \frac{d Y}{d T} T d T}{\int_{T_{\min }}^{T_{\max }} \frac{d Y}{d T} d T}$.

By integrating this equation between $T_{\min }$ and $T_{\max }$ we obtain a direct relation between $T_{\text {mean }}$ and $T_{\max }$ as a function of $\alpha$ and $c$ :

$T_{\text {mean }}=\frac{(1+1 / \alpha)\left(1-c^{1 / \alpha+2}\right)}{(2+1 / \alpha)\left(1-c^{1 / \alpha+1}\right)} T_{\max }$.

A comparison of the wdem model with the classical coolingflow model can be found in de Plaa et al. (2005). We note that the wdem model contains less cool gas than the classical cooling-flow model, which is consistent with recent observations (Peterson et al. 2001, 2003).

The spectral lines in the MEKAL model are fitted self consistently. From the fits we obtain the numbers of atoms of all elements with detected line emission. To see whether different elements show similar abundances with respect to solar, it is convenient to normalize these numbers with respect to the relative solar abundances. To make the comparison with previous work easier, we use the solar abundances as given by Anders \& Grevesse (1989) for this normalization. The more recent solar abundance determinations (e.g. Grevesse \& Sauval 1998; Lodders 2003) give significantly lower abundances of

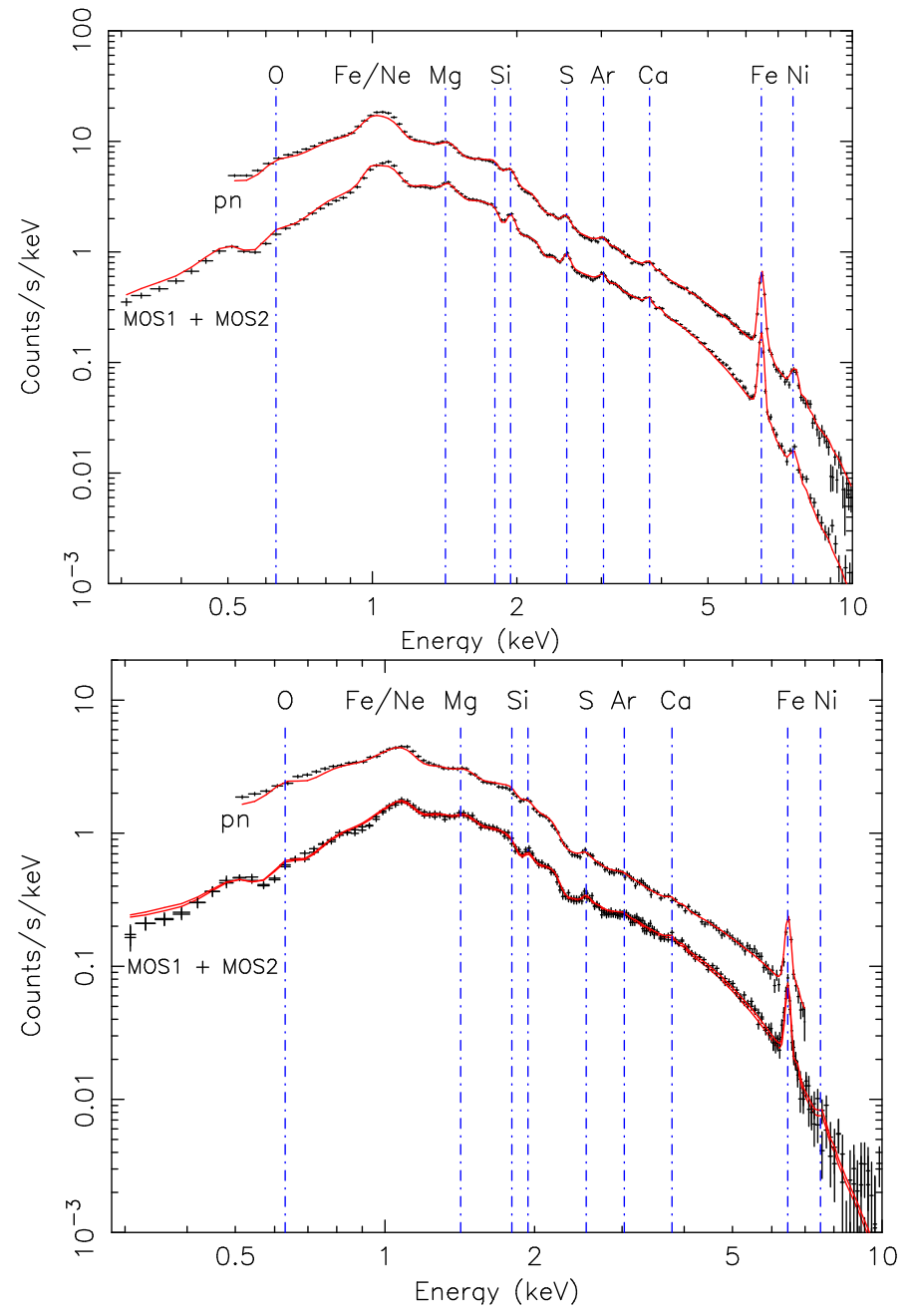

Fig. 1. The total spectrum of the core of the cluster (with a radius of $3^{\prime}$ ), which contains the cooling core (upper panel) and the spectrum of the $3^{\prime}-9^{\prime}$ region (lower panel). The continuous line represents the fitted wdem model. The EPIC pn and MOS1 + MOS2 spectra are indicated.

oxygen and neon than those measured by Anders \& Grevesse (1989). Use of these new determinations would only affect the representation of the elemental abundances in our paper, but not the actual measured values, which can be reconstructed by multiplication of the given values with the normalizations.

\section{Global spectrum}

\subsection{EPIC}

To compare the properties of the cluster in its cooling core region with the properties outside of the cooling core region we extract two spectra. One is from a circular region with a radius of $3^{\prime}$ centered on the X-ray maximum of the cluster and one is from an annulus with inner radius of $3^{\prime}$ and outer radius of $9^{\prime}$. The large temperature gradient and projection effects make it necessary to fit the spectra with a multi-temperature model. We use the wdem model, described in Sect. [3] and fix the abundances of elements with weak lines, which we do not fit, to 0.6 and 0.3 times solar in the inner and outer region respec- 
Table 2. Fit results obtained by fitting the wdem model to the spectrum extracted from a circular region with a radius of $3^{\prime}$ centered on the core of the cluster and from an annulus with inner and outer radii respectively $3^{\prime}$ and $9^{\prime}$. For the Galactic absorption we use a value of $N_{\mathrm{H}}=2.5 \times 10^{21} \mathrm{~cm}^{-2}$. Emission measures $\left(Y=\int n_{\mathrm{e}} n_{\mathrm{H}} d V\right)$ are given in $10^{66} \mathrm{~cm}^{-3}$. Abundances are given with respect to solar.

\begin{tabular}{l|cc}
\hline \hline Parameter & $0-3^{\prime}$ & $3-9^{\prime}$ \\
\hline$Y$ & $15.89 \pm 0.13$ & $6.53 \pm 0.01$ \\
$k T_{\max }(\mathrm{keV})$ & $3.75 \pm 0.02$ & $5.36 \pm 0.06$ \\
$\alpha$ & $0.75 \pm 0.02$ & $1.34 \pm 0.10$ \\
$k T_{\text {mean }}$ & $2.64 \pm 0.02$ & $3.46 \pm 0.05$ \\
$\mathrm{Mg}$ & $0.57 \pm 0.08$ & $0.21 \pm 0.06$ \\
$\mathrm{Si}$ & $0.72 \pm 0.03$ & $0.37 \pm 0.03$ \\
$\mathrm{~S}$ & $0.61 \pm 0.03$ & $0.27 \pm 0.04$ \\
$\mathrm{Ar}$ & $0.42 \pm 0.05$ & $0.14 \pm 0.09$ \\
$\mathrm{Ca}$ & $0.84 \pm 0.06$ & $0.62 \pm 0.12$ \\
$\mathrm{Fe}$ & $0.532 \pm 0.007$ & $0.383 \pm 0.006$ \\
$\mathrm{Ni}$ & $1.46 \pm 0.10$ & $0.77 \pm 0.15$ \\
\hline$\chi^{2} /$ d.o.f. & $948 / 525$ & $666 / 491$ \\
\hline
\end{tabular}

Table 3. Abundance upper limits for elements with weak lines, which could not be reliably detected, determined from the fluxes at the expected line energies of their helium-like emission. We also show the abundance of calcium calculated with the same method (it is consistent with the abundance determined in MEKAL).

\begin{tabular}{l|cc}
\hline \hline Element & $\mathrm{I}\left(\right.$ phot $\left.^{-2} \mathrm{~s}^{-1}\right)$ & Abund. (solar) \\
\hline $\mathrm{Ca}$ & $0.252 \pm 0.019$ & $0.80 \pm 0.05$ \\
$\mathrm{Ti}$ & $0.017 \pm 0.013$ & $1.5 \pm 1.1$ \\
$\mathrm{Cr}$ & $0.020 \pm 0.010$ & $0.5 \pm 0.2$ \\
$\mathrm{Mn}$ & $-0.003 \pm 0.009$ & $-0.2 \pm 0.5$ \\
$\mathrm{Co}$ & $0.000 \pm 0.008$ & $0 \pm 2$ \\
\hline
\end{tabular}

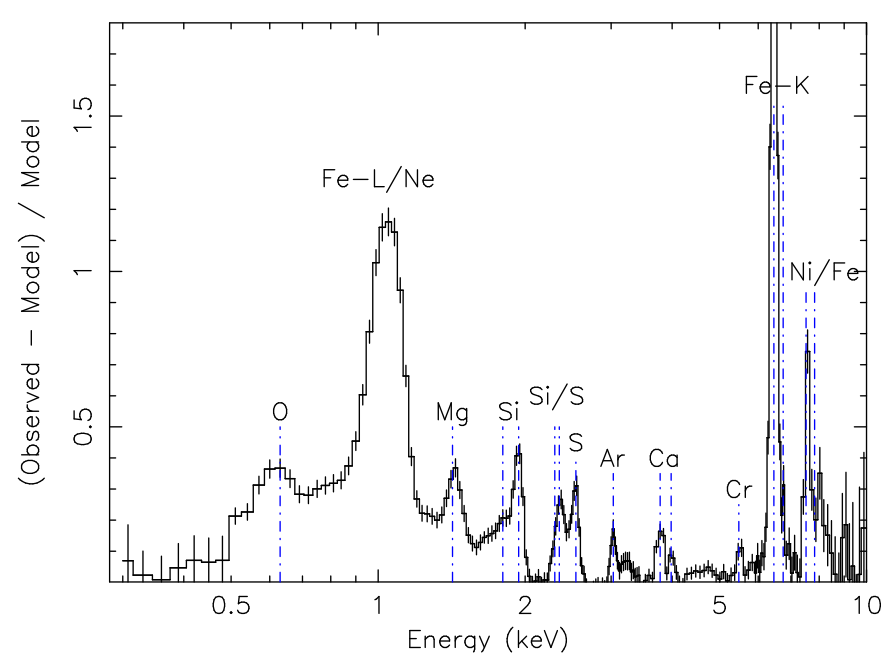

Fig. 2. Residuals of the fit to the EPIC total spectrum extracted from a circular region with a radius of $3^{\prime}$, with the line emission put to zero in the model. tively. The high statistics of the spectra allow us to determine the temperature structure and elemental abundances of several elements in these regions very precisely. The best-fit parameters are shown in Table 2

We find that the core is cooler than the outer region. We also find that the outer part has a broader temperature distribution than the core. The spectra with the indicated important spectral lines are shown in Fig. [1 All the detected spectral lines are shown in Fig. 2] which shows the residuals of the EPIC spectrum with line emission put to zero in the model, so all spectral lines are clearly visible. For the first time we see a feature at the expected energy of chromium in a cluster of galaxies, which corresponds to a detection with $2 \sigma$ significance. First, we attempt to measure the abundances of elements shown in Table 2

Since oxygen is predominantly produced by type II supernovae, it is an important element for constraining the enrichment scenarios. Oxygen has strong $\mathrm{H}$-like lines at $0.65 \mathrm{keV}$. The strong absorption toward 2A 0335+096 (see Appendix $\AA$, uncertainties in the calibration of EPIC and relatively nearby iron lines do not allow us to constrain the oxygen abundance accurately by EPIC. However, the RGS with its high spectral resolution allows us to determine the oxygen abundance in the core of the cluster (see Table 4). The $2 \mathrm{p}-1 \mathrm{~s}$ neon lines at 1.02 $\mathrm{keV}$ are in the middle of the iron L complex (lying between about 0.8 to $1.4 \mathrm{keV}$ ). The resolution of the EPIC cameras is not sufficient to resolve the individual lines in the iron L complex which makes the neon abundance determination by EPIC unreliable. However, the high resolution of RGS allows us to determine the neon abundance at least in the core of the cluster (see Table 4). The $\mathrm{K}$ shell lines of magnesium at $1.47 \mathrm{keV}$ are also close to the iron L complex, which makes our magnesium abundance determinations by EPIC somewhat sensitive to our temperature and iron abundance model. The magnesium abundance determinations at larger radii, where the surface brightness of the cluster is relatively low might be influenced by the instrumental aluminum line at $1.48 \mathrm{keV}$. The silicon, sulfur, argon and calcium lines lie in a relatively uncrowded part of the spectrum and their abundances are in general well determined. Iron has the strongest spectral lines in the X-ray band. At temperatures above $3 \mathrm{keV}$ its $\mathrm{K} \alpha$ lines are the strongest at about $6.67 \mathrm{keV}$ and $6.97 \mathrm{keV}$, while the iron L-shell complex ranging from about $0.8 \mathrm{keV}$ to $1.4 \mathrm{keV}$ dominates at lower temperatures. These spectral lines make the iron abundance determinations the most reliable of all elements. The nickel abundance is determined mainly from its K-shell line blends at $7.80 \mathrm{keV}$ and $8.21 \mathrm{keV}$, which are partially blended with iron lines.

In Table 2 we see that the abundances in the outer part are always lower than in the core of the cluster.

We also attempt to estimate the abundance upper limits for elements with weak lines (titanium, chromium, manganese, cobalt), which can not yet be fitted in MEKAL in a selfconsistent way. We determine their abundances from the fluxes at the expected line energies of their helium-like emission (see Table 3]. The feature at the expected energy of chromium corresponds to a detection at a $2 \sigma$ level, while the feature at the expected energy of titanium corresponds to a $1 \sigma$ detection. 


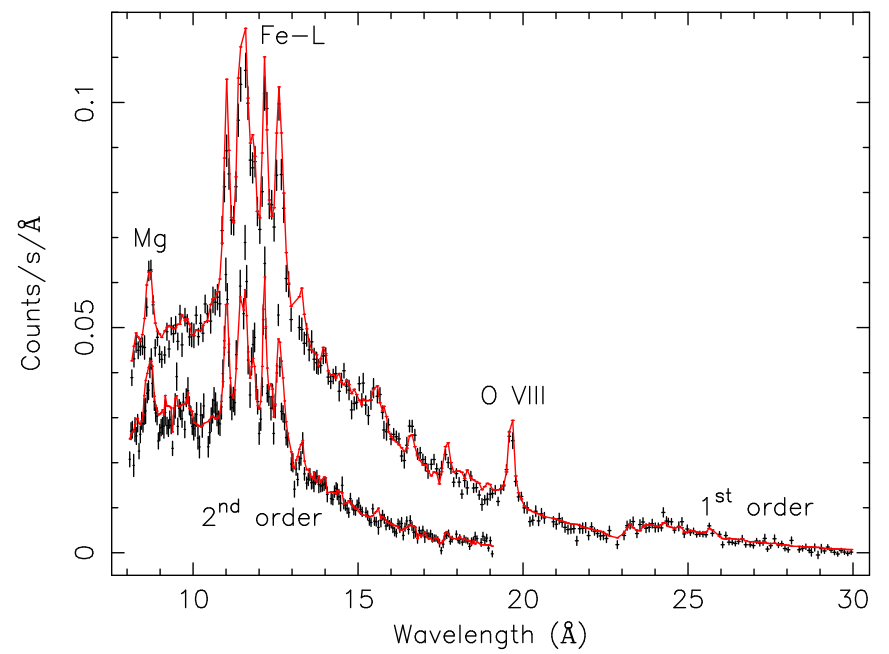

Fig. 3. $1^{\text {st }}$ and $2^{\text {nd }}$ order spectrum of $2 \mathrm{~A} 0335+096$ extracted from a $4^{\prime}$ wide strip centered on the core. The continuous line represents the fitted wdem model. Between 15 and $18 \AA$ $\mathrm{Fe}$ XVII and Fe XVIII emission lines are visible. On the $\mathrm{X}$-axis we show the observed wavelength.

Table 4. Fit results for the RGS spectra extracted from a $4^{\prime}$ wide strip. The value for $N_{\mathrm{H}}$ is in $10^{21} \mathrm{~cm}^{-2}$. Iron is given with respect to solar and the other abundances with respect to iron.

\begin{tabular}{l|cc}
\hline \hline Parameter & single-temp & wdem-model \\
\hline$N_{\mathrm{H}}$ & $3.14 \pm 0.03$ & $2.95 \pm 0.04$ \\
$k T(\mathrm{keV})$ & $1.80 \pm 0.02$ & \\
$k T_{\max }$ & & $3.36 \pm 0.13$ \\
$k T_{\text {mean }}$ & & $2.51 \pm 0.10$ \\
$\alpha$ & & $0.52 \pm 0.03$ \\
$\mathrm{O}_{\mathrm{abs}}$ & $0.47 \pm 0.02$ & $0.54 \pm 0.03$ \\
$\mathrm{~N} / \mathrm{Fe}$ & $1.7 \pm 0.6$ & $1.3 \pm 0.4$ \\
$\mathrm{O} / \mathrm{Fe}$ & $0.55 \pm 0.05$ & $0.49 \pm 0.05$ \\
$\mathrm{Ne} / \mathrm{Fe}$ & $1.11 \pm 0.10$ & $0.85 \pm 0.08$ \\
$\mathrm{Mg} / \mathrm{Fe}$ & $0.97 \pm 0.10$ & $0.97 \pm 0.08$ \\
$\mathrm{Fe}$ & $0.524 \pm 0.018$ & $1.07 \pm 0.06$ \\
$\mathrm{Scale} s_{O}$ & $0.59 \pm 0.12$ & $0.81 \pm 0.14$ \\
$\mathrm{Scale} s_{\mathrm{Fe}}$ & $0.60 \pm 0.04$ & $0.73 \pm 0.04$ \\
\hline$\chi^{2} /$ d.o.f. & $1552 / 790$ & $1173 / 788$ \\
\hline
\end{tabular}

\subsection{RGS}

In Fig. 3 we show the first and second order RGS spectra extracted from a $4^{\prime}$ wide strip in the cross-dispersion direction of the instrument. The extraction region is centered on the core of the cluster. Despite the spatial broadening, the strong spectral lines of magnesium, neon, iron and oxygen are well resolved in both spectral orders. In Table 4 we list the fit results for these spectra, for both the single temperature and wdem model.

The $\chi^{2}$ values of the fits show that the wdem model fits better than the single-temperature model. However, the $\chi^{2}$ for the wdem is still high, probably because of the disturbed nature of the core of the cluster. There are some systematic differences between the two models. The abundances found fitting the spectrum with the wdem model are in general twice the single-temperature values and the line widths of oxygen and iron, indicated with the scale parameters $s_{O}$ and $s_{F e}$, are also higher for wdem. Because of these differences, we divide the abundances of the other elements, by the value for iron. Since the continuum is not well determined by the RGS, these ratios are more reliable than the absolute value. The relative abundances, given in units with respect to solar, for neon and magnesium are similar to iron, while the oxygen abundance is about half the value for iron. We are also able, using the excellent statistics, to derive the abundance for nitrogen. This value is also consistent with that for iron, although the error bars are large. The widths of the oxygen and iron lines, indicated by the scale values, are very similar in this cluster, which shows that the spatial distribution of oxygen and iron might be similar.

Regarding the DEM temperature structure, the value of $\alpha$ $(0.52 \pm 0.03)$ for $w d e m$ is smaller than the EPIC value of $0.75 \pm$ 0.02 from within $3^{\prime}$ from the core, probably, because of the different extraction regions. The different extraction regions and the disturbed nature of the core (see sections 6 and 7 make the abundances determined from the EPIC and RGS difficult to compare. The mean temperature $k T_{\text {mean }}$ of the wdem results is $2.51 \pm 0.10 \mathrm{keV}$, which is higher than the single-temperature fit.

Because the solar oxygen abundance in Anders \& Grevesse (1989) is slightly overestimated, we need to free the oxygenabundance in the Galactic absorption component $\left(\mathrm{O}_{a b s}\right)$ in our spectral model. This effect in absorption was first observed by Weisskopf et al. (2004) in an observation of the Crab pulsar and later confirmed in a cluster observation of Abell 478 by de Plaa et al. (2004). The values of $0.47 \pm 0.02$ and $0.54 \pm 0.03$ that we find for the oxygen-abundance in the Galactic absorption component $\mathrm{O}_{\mathrm{abs}}$ are slightly lower than the RGS values found by de Plaa et al. (2004), but consistent with the expected value of $0.58 \pm 0.08$ based on an updated value for the solar oxygen abundance by Allende Prieto et al. (2001).

\section{Radial profiles}

We determine the radial temperature and abundance profiles of several elements using projected and deprojected spectra extracted from circular annuli, centered on the X-ray maximum of the cluster. We use annuli with outer radii indicated on the top of Table 5 We also extract radial temperature and abundance profiles from RGS in the cross-dispersion direction.

\subsection{Projected spectra}

We fit the spectra extracted from the annuli with three different models, and compare the results. Due to high background in the 6th and 7th annulus we ignore the pn data at energies higher than $7.5 \mathrm{keV}$. In our model we fix the abundances of elements which we do not fit to 0.3 solar. The results are presented in Fig. 4 and Table 5 First we fit the spectra with a single temperature model. We find that the model fits our data poorly and the $\chi^{2}$ in the core of the cluster is unacceptably high. Based on the large $\chi^{2}$ of the fit, in the central region we can discard the single temperature model. We therefore try to fit 2 thermal models simultaneously, with coupled abundances and with a fixed separation between the two temperatures as $T_{2}=2 T_{1}$ (where $T_{1}$ 

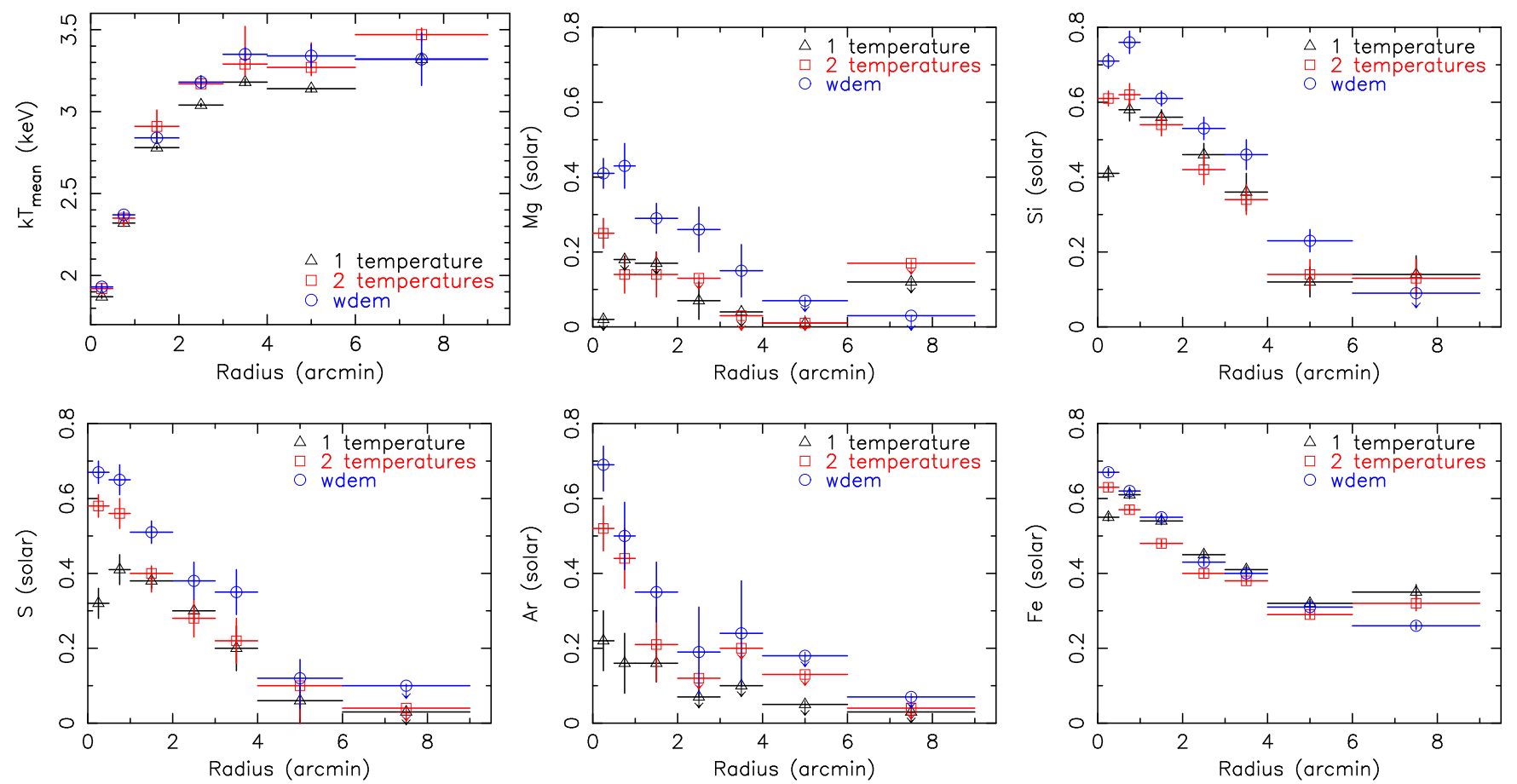

Fig. 4. Comparison of fit results obtained by fitting the EPIC data with a single-temperature model, two-temperature model and the wdem model.

and $T_{2}$ are the temperatures of the two thermal components). Fixing the temperature to $T_{2}=2 T_{1}$ is a good first approximation of the multi-temperature structure, since Kaastra et al. (2004) and Peterson et al. (2003) found that in almost all cases of their cluster samples, at each radius, there is negligible emission from gas with a temperature less than one-third to half of the fitted upper temperature. Using the two temperature model our fits improve significantly. The $\chi^{2}$ improves most significantly in the core of the cluster, but we can see a slight improvement also at outer radii. However, fitting the data with the wdem model further improves the $\chi^{2}$ of our fits in 6 of 7 extraction annuli.

Fitting the data with a single-temperature thermal model we see that the abundances of all elements except argon drop in the core of the cluster and have a off-center peak. As we go from the single-temperature model to the two-temperature model and further to the wdem model, abundances of all elements in general increase, especially in the central regions of the cluster. The wdem model shows that most of the abundances peak in the core of the cluster, we detect a slight hint of a drop in the center only for calcium, silicon and magnesium. The dependence of the iron abundance on the temperature model is known as the Fe-bias (see, e.g. Buote 2000; Molendi \& Gastaldello 2001). As we go from one model to the other, the abundance value of magnesium varies the most. Due to its vicinity to the Fe- $\mathrm{L}$ complex its abundance is very sensitive to the temperature and iron abundance model.

In general, all three models show a drop of abundances toward the outer parts of the cluster.

\subsection{Deprojected spectra}

To account for projection effects in the core of the cluster we extract spectra, deprojected under the assumption of a spherical symmetry. We use the background event files of Lumb et al. (2002). We extract the deprojected spectra from 5 annuli in the inner $0^{\prime}-4^{\prime}$, where the cluster is still bright enough and, as shown in Appendix $\mathrm{A}$ the background subtraction does not have a significant influence on our results. The deprojected spectra represent the count rates from spherical shells centered on the core of the cluster. Our extraction method and data analysis for the deprojected spectra is described extensively in Kaastra et al. (2004).

The fit results for the deprojected spectra are shown in Table 6 The temperatures determined from deprojected and projected spectra are compared in Fig. 5 Since, in the process of deprojection we account for the hot plasma lying in front of the relatively cool core, the observed temperatures of the deprojected shells are lower than those determined without deprojection. While the $\chi^{2}$ of the fit of the single-temperature model improves significantly if we deproject the spectrum (compare with the $\chi^{2}$ in Table 5), the single temperature thermal model still does not describe our spectra well. The wdem model describes the deprojected spectra extracted from the core of the cluster significantly better than the single-temperature model. It shows, that the temperature gradients in the extraction regions and the intrinsic multi-temperature structure make it necessary to use multi-temperature models also when fitting deprojected spectra. Although the deprojected spectra give a better value for the temperature, with the deprojection we introduce more noise into the spectra so the determination of abundances becomes more uncertain. 
Table 5. Fit results obtained by fitting the EPIC data with a single-temperature model (1), two-temperature model (2) and the wdem model (3). Emission measures $\left(Y=\int n_{\mathrm{e}} n_{\mathrm{H}} d V\right)$ are given in $10^{66} \mathrm{~cm}^{-3}$. Abundances are given with respect to solar.

\begin{tabular}{|c|c|c|c|c|c|c|c|c|}
\hline & Model & $0-0.5^{\prime}$ & $0.5-1.0^{\prime}$ & $1.0-2.0^{\prime}$ & $2.0-3.0^{\prime}$ & $3.0-4.0^{\prime}$ & $4.0-6.0^{\prime}$ & $6.0-9.0^{\prime}$ \\
\hline \multirow[t]{3}{*}{$\bar{Y}$} & 1 & 3.55 & 4.65 & 5.22 & 3.19 & 2.01 & 2.85 & 2.97 \\
\hline & 2 & 3.42 & 4.82 & 5.45 & 3.31 & 2.08 & 2.75 & 2.56 \\
\hline & 3 & 3.21 & 4.52 & 5.30 & 3.26 & 2.05 & 2.85 & 2.23 \\
\hline$k T(\mathrm{keV})$ & 1 & $1.87 \pm 0.01$ & $2.32 \pm 0.01$ & $2.78 \pm 0.01$ & $3.04 \pm 0.01$ & $3.18 \pm 0.02$ & $3.14 \pm 0.02$ & $3.32 \pm 0.04$ \\
\hline$k T_{2}(\mathrm{keV})$ & 2 & $2.154_{-0.002}^{+0.005}$ & $2.72 \pm 0.04$ & $3.51 \pm 0.09$ & $3.08 \pm 0.01$ & $3.92_{-0.07}^{+0.23}$ & $4.10_{-0.05}^{+0.15}$ & $4.39 \pm 0.04$ \\
\hline$k T_{\max }(\mathrm{keV})$ & 3 & $2.64 \pm 0.02$ & $3.14 \pm 0.02$ & $3.95 \pm 0.03$ & $4.39 \pm 0.04$ & $4.81 \pm 0.05$ & $4.97 \pm 0.05$ & $5.84 \pm 0.14$ \\
\hline$k T_{\text {mean }}(\mathrm{keV})$ & 3 & $1.93 \pm 0.02$ & $2.37 \pm 0.02$ & $2.84 \pm 0.03$ & $3.18 \pm 0.04$ & $3.35 \pm 0.04$ & $3.34 \pm 0.08$ & $3.32 \pm 0.16$ \\
\hline$\alpha$ & 3 & $0.58 \pm 0.01$ & $0.48 \pm 0.02$ & $0.65 \pm 0.02$ & $0.62 \pm 0.04$ & $0.79 \pm 0.04$ & $1.01_{-0.02}^{+0.23}$ & $8.02_{-3}^{+13}$ \\
\hline \multirow[t]{3}{*}{$\mathrm{Mg}$} & 1 & $<0.02$ & $<0.18$ & $0.17 \pm 0.03$ & $0.07 \pm 0.05$ & $<0.04$ & $<0.01$ & $<0.12$ \\
\hline & 2 & $0.25 \pm 0.04$ & $0.14 \pm 0.05$ & $0.14 \pm 0.06$ & $<0.13$ & $<0.03$ & $<0.01$ & $<0.17$ \\
\hline & 3 & $0.41 \pm 0.04$ & $0.43 \pm 0.06$ & $0.29 \pm 0.04$ & $0.26 \pm 0.06$ & $0.15 \pm 0.07$ & $<0.07$ & $<0.03$ \\
\hline \multirow[t]{3}{*}{$\mathrm{Si}$} & 1 & $0.41 \pm 0.02$ & $0.58 \pm 0.03$ & $0.56 \pm 0.02$ & $0.46 \pm 0.03$ & $0.36 \pm 0.05$ & $0.12 \pm 0.04$ & $0.14 \pm 0.05$ \\
\hline & 2 & $0.61 \pm 0.02$ & $0.62 \pm 0.03$ & $0.54 \pm 0.03$ & $0.42 \pm 0.04$ & $0.34 \pm 0.04$ & $0.14 \pm 0.04$ & $0.13 \pm 0.05$ \\
\hline & 3 & $0.71 \pm 0.02$ & $0.76 \pm 0.03$ & $0.61 \pm 0.02$ & $0.53 \pm 0.03$ & $0.46 \pm 0.04$ & $0.23 \pm 0.03$ & $<0.09$ \\
\hline \multirow[t]{3}{*}{$\mathrm{S}$} & 1 & $0.32 \pm 0.04$ & $0.41 \pm 0.04$ & $0.38 \pm 0.02$ & $0.30 \pm 0.02$ & $0.20 \pm 0.06$ & $<0.06$ & $<0.03$ \\
\hline & 2 & $0.58 \pm 0.03$ & $0.56 \pm 0.04$ & $0.40 \pm 0.04$ & $0.28 \pm 0.05$ & $0.22 \pm 0.06$ & $<0.10$ & $<0.04$ \\
\hline & 3 & $0.67 \pm 0.03$ & $0.65 \pm 0.04$ & $0.51 \pm 0.03$ & $0.38 \pm 0.05$ & $0.35 \pm 0.06$ & $0.12 \pm 0.07$ & $<0.10$ \\
\hline \multirow[t]{3}{*}{$\mathrm{Ar}$} & 1 & $0.22 \pm 0.08$ & $0.16 \pm 0.08$ & $0.16 \pm 0.05$ & $<0.07$ & $<0.10$ & $<0.05$ & $<0.03$ \\
\hline & 2 & $0.52 \pm 0.06$ & $0.44 \pm 0.08$ & $0.21 \pm 0.10$ & $<0.12$ & $<0.20$ & $<0.13$ & $<0.04$ \\
\hline & 3 & $0.69 \pm 0.06$ & $0.50 \pm 0.09$ & $0.35 \pm 0.08$ & $0.19 \pm 0.12$ & $0.24 \pm 0.14$ & $<0.18$ & $<0.07$ \\
\hline \multirow[t]{3}{*}{$\mathrm{Ca}$} & 1 & $0.51 \pm 0.12$ & $0.66 \pm 0.10$ & $0.85 \pm 0.07$ & $0.68 \pm 0.09$ & $0.53 \pm 0.15$ & $0.57 \pm 0.15$ & $0.27 \pm 0.21$ \\
\hline & 2 & $0.66 \pm 0.09$ & $0.96 \pm 0.10$ & $0.92 \pm 0.12$ & $0.71 \pm 0.16$ & $0.66 \pm 0.14$ & $0.70 \pm 0.14$ & $0.30 \pm 0.23$ \\
\hline & 3 & $0.74 \pm 0.09$ & $0.98 \pm 0.11$ & $1.02 \pm 0.10$ & $0.77 \pm 0.15$ & $0.74 \pm 0.17$ & $0.66 \pm 0.17$ & $0.70 \pm 0.23$ \\
\hline \multirow[t]{3}{*}{$\mathrm{Fe}$} & 1 & $0.55 \pm 0.01$ & $0.61 \pm 0.01$ & $0.54 \pm 0.01$ & $0.45 \pm 0.01$ & $0.41 \pm 0.01$ & $0.32 \pm 0.01$ & $0.35 \pm 0.02$ \\
\hline & 2 & $0.63 \pm 0.01$ & $0.57 \pm 0.01$ & $0.48 \pm 0.01$ & $0.40 \pm 0.01$ & $0.38 \pm 0.01$ & $0.29 \pm 0.01$ & $0.32 \pm 0.02$ \\
\hline & 3 & $0.67 \pm 0.01$ & $0.62 \pm 0.01$ & $0.55 \pm 0.01$ & $0.43 \pm 0.01$ & $0.40 \pm 0.01$ & $0.31 \pm 0.01$ & $0.26 \pm 0.01$ \\
\hline \multirow[t]{3}{*}{$\mathrm{Ni}$} & 1 & $0.68 \pm 0.11$ & $1.08 \pm 0.13$ & $0.57 \pm 0.08$ & $0.35 \pm 0.11$ & $0.37 \pm 0.20$ & un. & un. \\
\hline & 2 & $0.89 \pm 0.09$ & $0.90 \pm 0.12$ & $0.53 \pm 0.13$ & $0.29 \pm 0.15$ & $0.22 \pm 0.17$ & un. & un. \\
\hline & 3 & $1.65 \pm 0.10$ & $1.52 \pm 0.14$ & $0.99 \pm 0.10$ & $0.64 \pm 0.20$ & $0.69 \pm 0.17$ & un. & un. \\
\hline \multirow[t]{3}{*}{$\chi^{2} /$ d.o.f. } & 1 & $2444 / 525$ & $1278 / 525$ & $1066 / 525$ & $774 / 525$ & $717 / 525$ & $909 / 499$ & $816 / 476$ \\
\hline & 2 & $844 / 525$ & $753 / 525$ & $883 / 525$ & $680 / 525$ & $664 / 525$ & $789 / 499$ & $742 / 476$ \\
\hline & 3 & $782 / 525$ & $664 / 525$ & $707 / 525$ & $596 / 525$ & $532 / 525$ & $607 / 499$ & $880 / 476$ \\
\hline
\end{tabular}

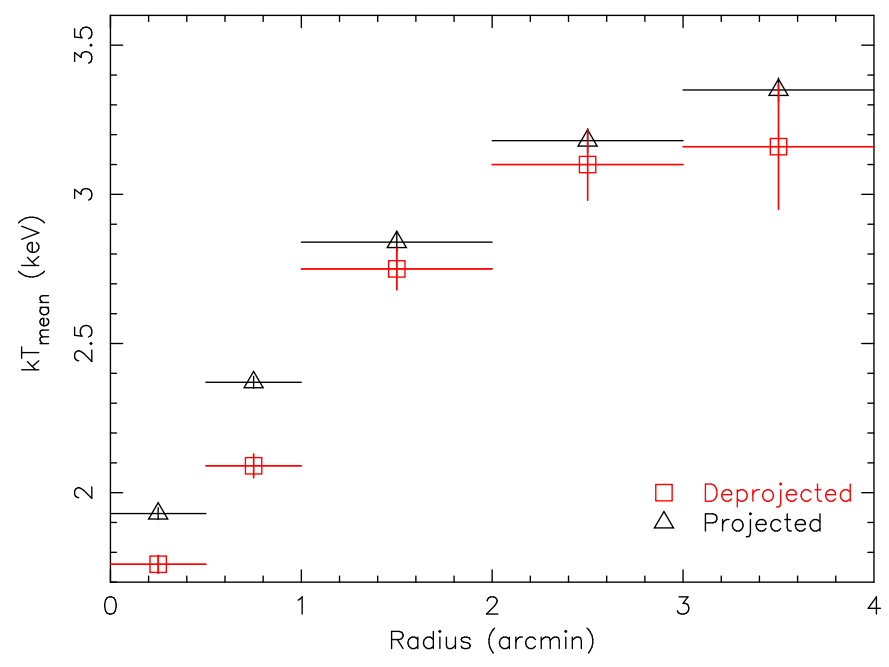

Fig. 5. Comparison of the projected and deprojected mean temperatures obtained by fitting the wdem model.

\subsection{RGS radial profiles}

Because of the high statistics of the RGS spectrum shown in Fig. 3] we are also able to extract smaller strips in the crossdispersion direction and make a radial profile up to $2^{\prime}$ from the center. The results of these fits are shown in Table[7 It confirms the radial temperature profile in the core found with EPIC, although the temperature determined with RGS is slightly higher. The reason for this discrepancy is probably the difference in extraction regions. The RGS spectra also contain a small contribution of photons originating outside the core radius due to the spatial extent of the source. Again, like in the full fieldof-view case, the mean temperatures $k T_{\text {mean }}$ are slightly higher than the single-temperature fits, although the values are consistent within the error-bars. The value for $\alpha$ is rather well constrained in the region between $-2^{\prime}$ and $0.5^{\prime}$ and shows a slight discontinuity around $0^{\prime}$, but the statistics do not allow to draw a conclusion. From the $\chi^{2}$ values it is clear that a DEM model (wdem) fits the RGS spectra better.

From the RGS abundance profiles in the cross-dispersion direction, which are listed in Table 7 we confirm the spatial distribution of oxygen and iron indicated by the line widths 
Table 6. Fit results of the deprojected EPIC spectra extracted from 5 annuli in the inner $4^{\prime}$ and fitted with a single-temperature model (1) and wdem model (3). This region contains the cooling core, where the projection effects are the most important. Abundances are given with respect to solar.

\begin{tabular}{l|c|ccccc}
\hline \hline Parameter & Model & $0-0.5^{\prime}$ & $0.5-1.0^{\prime}$ & $1.0-2.0^{\prime}$ & $2.0-3.0^{\prime}$ & $3.0-4.0^{\prime}$ \\
\hline$N_{\mathrm{H}}\left(10^{21} \mathrm{~cm}^{-2}\right)$ & 1 & $2.36 \pm 0.02$ & $2.37 \pm 0.02$ & $2.47 \pm 0.03$ & $2.48 \pm 0.05$ & $2.53 \pm 0.05$ \\
& 3 & $2.52 \pm 0.06$ & $2.58 \pm 0.05$ & $2.64 \pm 0.08$ & $2.64 \pm 0.11$ & $2.66 \pm 0.16$ \\
\hline$k T(\mathrm{keV})$ & 1 & $1.65 \pm 0.02$ & $2.07 \pm 0.02$ & $2.68 \pm 0.02$ & $3.04 \pm 0.03$ & $3.11 \pm 0.05$ \\
$k T_{\max }(\mathrm{keV})$ & 3 & $2.36 \pm 0.04$ & $2.72 \pm 0.04$ & $3.65 \pm 0.08$ & $4.28 \pm 0.13$ & $4.25 \pm 0.21$ \\
$k T_{\text {mean }}(\mathrm{keV})$ & 3 & $1.76 \pm 0.03$ & $2.09 \pm 0.04$ & $2.75 \pm 0.07$ & $3.10 \pm 0.12$ & $3.16 \pm 0.21$ \\
\hline$\alpha$ & 3 & $0.52 \pm 0.03$ & $0.43 \pm 0.03$ & $0.49 \pm 0.04$ & $0.62 \pm 0.08$ & $0.53 \pm 0.14$ \\
& 3 & $0.6 \pm 0.2$ & $0.7 \pm 0.2$ & $0.3 \pm 0.2$ & $0.3 \pm 0.2$ & $<0.5$ \\
\hline $\mathrm{Mg}$ & 1 & $0.11 \pm 0.08$ & $0.34 \pm 0.10$ & $0.65 \pm 0.07$ & $0.35 \pm 0.13$ & $<0.16$ \\
& 3 & $0.55 \pm 0.10$ & $0.53 \pm 0.11$ & $0.76 \pm 0.14$ & $0.36 \pm 0.15$ & $<0.26$ \\
\hline $\mathrm{Si}$ & 1 & $0.46 \pm 0.04$ & $0.73 \pm 0.05$ & $0.80 \pm 0.04$ & $0.73 \pm 0.07$ & $0.52 \pm 0.09$ \\
& 3 & $0.74 \pm 0.06$ & $0.84 \pm 0.06$ & $0.84 \pm 0.07$ & $0.71 \pm 0.08$ & $0.50 \pm 0.10$ \\
\hline $\mathrm{S}$ & 1 & $0.42 \pm 0.04$ & $0.61 \pm 0.05$ & $0.52 \pm 0.04$ & $0.47 \pm 0.08$ & $0.19 \pm 0.12$ \\
& 3 & $0.66 \pm 0.06$ & $0.75 \pm 0.06$ & $0.61 \pm 0.07$ & $0.51 \pm 0.09$ & $0.25 \pm 0.10$ \\
\hline $\mathrm{Ar}$ & 1 & $0.40 \pm 0.11$ & $0.50 \pm 0.11$ & $0.25 \pm 0.10$ & $<0.14$ & $0.3 \pm 0.2$ \\
& 3 & $0.57 \pm 0.14$ & $0.69 \pm 0.12$ & $0.33 \pm 0.15$ & $<0.21$ & $0.4 \pm 0.3$ \\
\hline $\mathrm{Ca}$ & 1 & $0.64 \pm 0.20$ & $0.81 \pm 0.18$ & $1.00 \pm 0.13$ & $0.9 \pm 0.2$ & $0.4 \pm 0.3$ \\
& 3 & $0.41 \pm 0.20$ & $0.88 \pm 0.16$ & $1.06 \pm 0.18$ & $1.0 \pm 0.3$ & $0.5 \pm 0.4$ \\
\hline $\mathrm{Fe}$ & 1 & $0.50 \pm 0.02$ & $0.65 \pm 0.02$ & $0.61 \pm 0.02$ & $0.46 \pm 0.02$ & $0.41 \pm 0.03$ \\
& 3 & $0.67 \pm 0.03$ & $0.66 \pm 0.03$ & $0.57 \pm 0.02$ & $0.45 \pm 0.02$ & $0.40 \pm 0.03$ \\
\hline $\mathrm{Ni}$ & 1 & $0.62 \pm 0.17$ & $1.8 \pm 0.2$ & $1.53 \pm 0.18$ & $0.9 \pm 0.4$ & $0.6 \pm 0.5$ \\
& 3 & $1.3 \pm 0.3$ & $2.0 \pm 0.3$ & $1.8 \pm 0.3$ & $0.8 \pm 0.4$ & $0.6 \pm 0.5$ \\
\hline$\chi^{2} /$ d.o.f. & 1 & $1090 / 587$ & $668 / 570$ & $892 / 587$ & $515 / 587$ & $520 / 587$ \\
& 3 & $622 / 587$ & $489 / 587$ & $526 / 587$ & $481 / 587$ & $515 / 587$ \\
\hline & & & & & &
\end{tabular}

Table 7. Fit results for spatially resolved RGS spectra between 8-25 A. The fitted models are (1) single-temperature CIE and (3) $w d e m$. We use $2 \sigma$ upper limits.

\begin{tabular}{l|c|cccccc}
\hline \hline Parameter & Model & $-2.0 /-1.0^{\prime}$ & $-1.0 /-0.5^{\prime}$ & $-0.5 / 0^{\prime}$ & $0 / 0.5^{\prime}$ & $0.5-1.0^{\prime}$ & $1.0-2.0^{\prime}$ \\
\hline$k T(\mathrm{keV})$ & 1 & $4.12 \pm 0.13$ & $3.37 \pm 0.10$ & $2.89 \pm 0.06$ & $2.53 \pm 0.05$ & $3.21 \pm 0.12$ & $4.4 \pm 0.3$ \\
$k T_{\max }$ & 3 & $6.6 \pm 0.3$ & $5.2 \pm 0.2$ & $4.6 \pm 0.2$ & $3.73 \pm 0.14$ & $5.3 \pm 0.8$ & $7.8 \pm 0.9$ \\
$k T_{\text {mean }}$ & 3 & $4.6 \pm 0.2$ & $3.70 \pm 0.15$ & $3.12 \pm 0.15$ & $2.72 \pm 0.11$ & $3.8 \pm 0.7$ & $5.1 \pm 0.6$ \\
\hline$\alpha$ & 3 & $0.79 \pm 0.08$ & $0.70 \pm 0.06$ & $0.94 \pm 0.10$ & $0.60 \pm 0.05$ & $0.7 \pm 0.4$ & $1.3 \pm 0.3$ \\
\hline $\mathrm{O} / \mathrm{Fe}$ & 1 & $0.28 \pm 0.07$ & $0.34 \pm 0.05$ & $0.40 \pm 0.04$ & $0.36 \pm 0.04$ & $0.35 \pm 0.07$ & $0.57 \pm 0.18$ \\
& 3 & $0.29 \pm 0.06$ & $0.34 \pm 0.05$ & $0.41 \pm 0.04$ & $0.36 \pm 0.04$ & $0.36 \pm 0.06$ & $0.47 \pm 0.14$ \\
\hline $\mathrm{Ne} / \mathrm{Fe}$ & 1 & $1.34 \pm 0.17$ & $1.34 \pm 0.15$ & $1.83 \pm 0.14$ & $1.54 \pm 0.12$ & $1.00 \pm 0.17$ & $1.4 \pm 0.5$ \\
& 3 & $0.60 \pm 0.15$ & $0.73 \pm 0.13$ & $0.98 \pm 0.10$ & $0.98 \pm 0.10$ & $0.56 \pm 0.15$ & $<0.3$ \\
\hline $\mathrm{Mg} / \mathrm{Fe}$ & 1 & $<0.4$ & $0.66 \pm 0.17$ & $0.40 \pm 0.12$ & $<0.4$ & $<0.5$ & $<0.2$ \\
& 3 & $0.5 \pm 0.2$ & $1.19 \pm 0.17$ & $1.09 \pm 0.12$ & $0.68 \pm 0.11$ & $0.42 \pm 0.18$ & $1.0 \pm 0.5$ \\
\hline $\mathrm{Scale} s$ & 1 & $1.5 \pm 0.2$ & $0.98 \pm 0.10$ & $1.01 \pm 0.10$ & $0.90 \pm 0.07$ & $0.77 \pm 0.13$ & $4.5 \pm 1.0$ \\
& 3 & $1.30 \pm 0.16$ & $0.78 \pm 0.10$ & $0.69 \pm 0.06$ & $0.62 \pm 0.05$ & $0.58 \pm 0.11$ & $3.0 \pm 0.7$ \\
\hline$\chi^{2} /$ d.o.f. & 1 & $852 / 701$ & $977 / 701$ & $1491 / 701$ & $1337 / 701$ & $932 / 701$ & $923 / 701$ \\
& 3 & $757 / 698$ & $802 / 698$ & $800 / 698$ & $853 / 698$ & $851 / 698$ & $839 / 698$ \\
\hline
\end{tabular}

in the full RGS spectrum. The $\mathrm{O} / \mathrm{Fe}$ ratio is consistent with a value between $0.3-0.4$ over the whole profile. The neon and magnesium abundance tend to peak in the center slightly more than iron. Because the individual spectra in the RGS spatial profile have lower statistics than the total RGS spectrum, we use one scale parameter $s$ for all measured lines. Therefore, the fitted scale parameter, which accounts for the broadening of the lines because of the spatial extend of the source, is the average of all line widths given in units of the width of cluster emission.
These widths increase naturally toward the outer part of the cluster, because the spatial profile of the cluster also flattens.

\section{Temperature and iron abundance maps}

In order to investigate the spatial variations of the temperature and metallicity we extract temperature and iron abundance maps of the $5.5^{\prime} \times 5.5^{\prime}$ region centered on the core of the cluster.

First, we determine the temperature and iron abundance on a grid with bin-size of $30^{\prime \prime} \times 30^{\prime \prime}$. For every bin we compute 

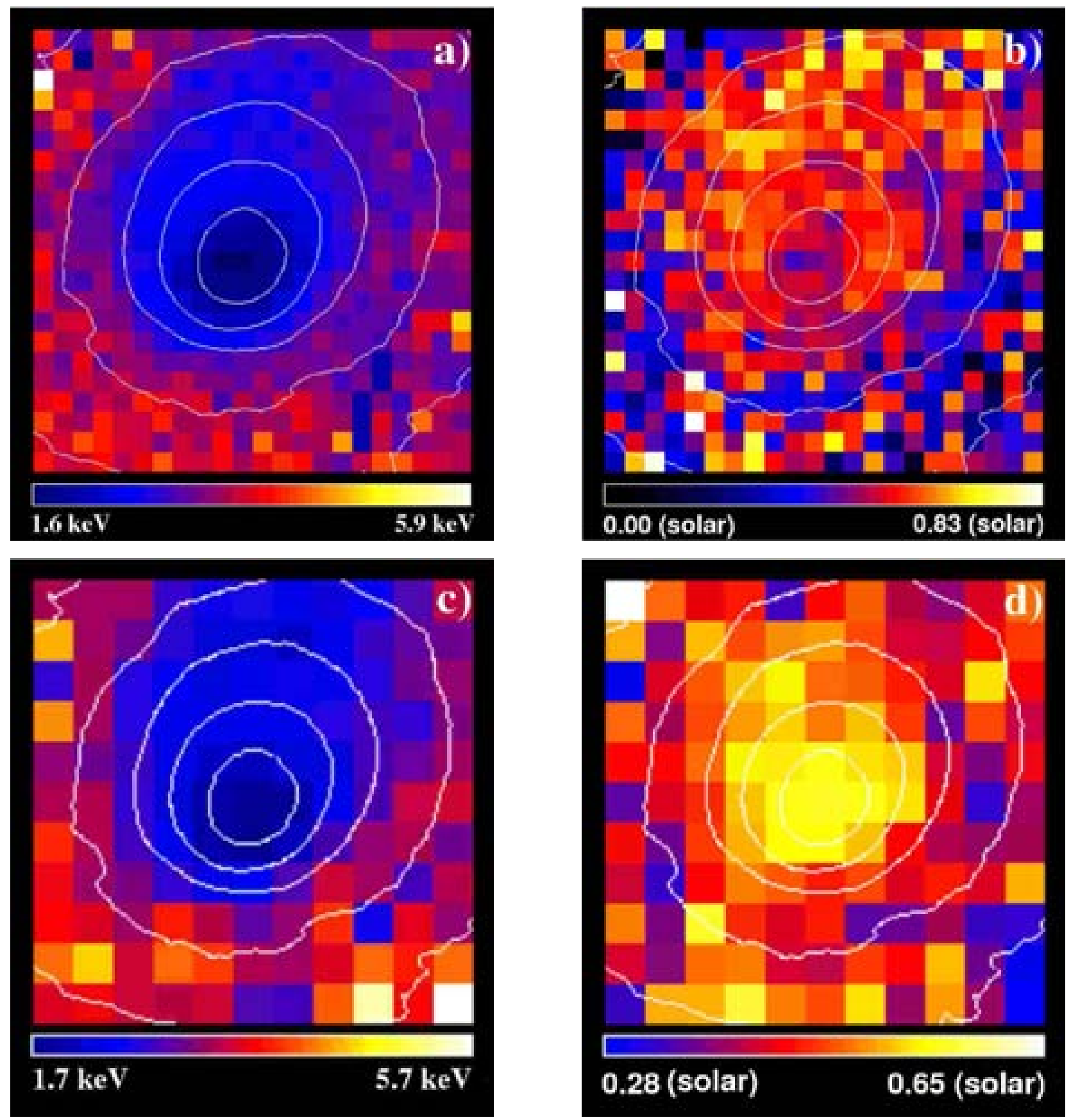

Fig. 6. Temperature and iron abundance maps of the inner $5.5^{\prime} \times 5.5^{\prime}$ region of the cluster. Panel $a$ and $b$ : temperature and iron abundance map with each $15^{\prime \prime} \times 15^{\prime \prime}$ pixel fitted with a single-temperature model. Panel $c$ and $d$ : mean temperature and iron abundance map with each $30^{\prime \prime} \times 30^{\prime \prime}$ pixel fitted with a wdem model. Note that North is up, West is to the right. All maps have overplotted the same X-ray isophots.

a redistribution and an ancillary file. We fit the spectrum of each bin individually by the multi-temperature wdem model. The spectral fits are done with a hydrogen column density fixed to the global value $2.5 \times 10^{21} \mathrm{~cm}^{-2}$ (see Appendix A. The redshift is fixed to the spectroscopically determined value of the central cD galaxy $(z=0.0349)$. The abundances of all elements except iron in our model are fixed to 0.3 times the solar value (the exact value of these abundances does not influence the temperature and iron abundance determinations), while the iron abundance is left as a free parameter. We fix the param- eter $\alpha$ to 0.58 , which is the value determined for the core of the cluster (see Table 5). The free parameters are the maximum temperature $T_{\max }$, the iron abundance and the normalization of the wdem component.

The temperature map, which shows the mean temperature $T_{\text {mean }}$ of the wdem model, reveals an elongated temperature structure in the South-Southeast North-Northwest direction and a sharp temperature change over the brightness edge south of the core. The iron abundance map shows a highly centrally 


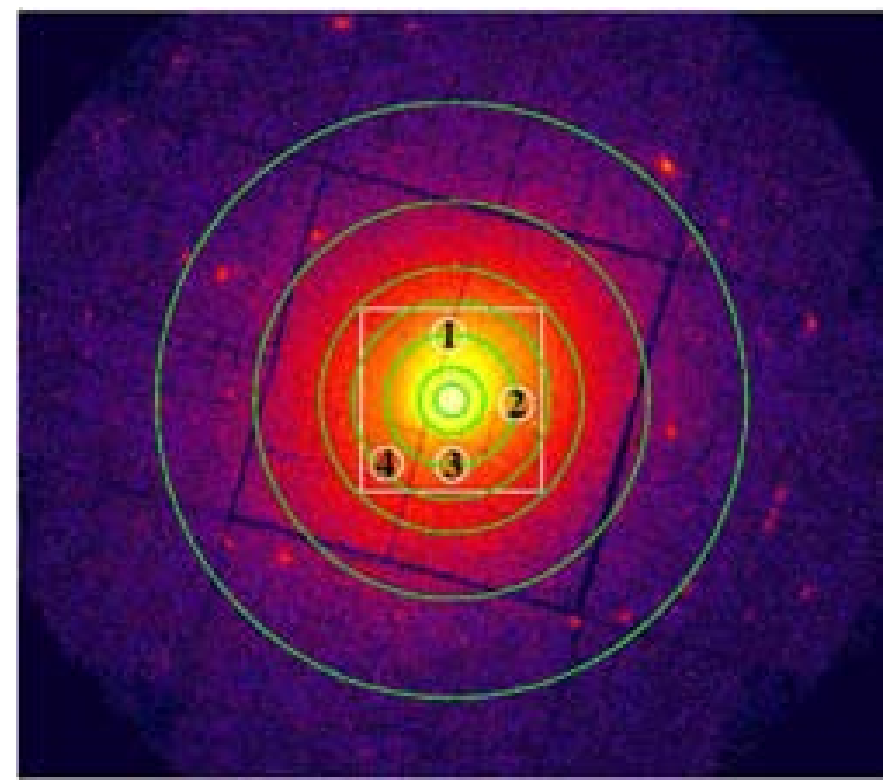

Fig. 7. MOS1 + MOS2 image of 2A 0335+096 with overplotted extraction regions. The concentric circles correspond to our extraction annuli used to investigate the radial profiles, the square shows the $5.5^{\prime} \times 5.5^{\prime}$ region used to extract the temperature and iron abundance maps and the small circles correspond to extraction regions $1,2,3$ and 4 used to verify that the temperature and abundances vary with position angle.

peaked iron abundance with an extension to the North (see the panel $c$ and $d$ in Fig. 6).

High count rates in the $5.5^{\prime} \times 5.5^{\prime}$ central region allow us to reduce the size of the bins to $15^{\prime \prime} \times 15^{\prime \prime}$ (which is roughly the FWHM of the point spread function of the pn detector) and extract a higher resolution temperature and iron abundance map fitting a single temperature model to each bin (see panel $a$ and $b$ in Fig. 6). The map shows a sharp temperature change on the southern side of the core and a large extension of the cool temperature gas to North-Northwest. In contrast to the wdem model fit, the iron abundance map extracted using a singletemperature model shows a central drop of iron abundance, with an abundance peak in a "high metallicity ring" around the $\mathrm{X}$-ray core with an extension to the North. We verified that the central drop of iron abundance is evident also in a temperature map with $30^{\prime \prime} \times 30^{\prime \prime}$ bins fitted with a single temperature model. The central abundance drop is rather the result of the oversimplified model for the temperature structure of the core of the cluster, than a real feature (see subsection 5.1.

To verify the azimuthal differences in the temperature and the metallicity we extract spectra from 4 circular regions with a radius of $0.5^{\prime}$ and fit them with the wdem model. The extraction regions are shown in Fig. (7) The regions 1, 2 and 3 are centered at the same distance from the core $\left(2^{\prime}\right)$ but at different position angles (North, West, South). Region 4 is at a distance of 2.75' from the core at South-Southeast. Apart from the wdem normalization, $T_{\max }$ and the iron abundance we leave free and fit the parameter $\alpha, N_{\mathrm{H}}$ and the silicon and sulfur abundance. The best-fit parameter values are given in Table 8 We confirm the
Table 8. Parameter values obtained by fitting regions 1, 2, 3 and 4 indicated in Fig. 7 with the wdem model. Emission measures $\left(Y=\int n_{\mathrm{e}} n_{\mathrm{H}} d V\right)$ are given in $10^{66} \mathrm{~cm}^{-3}$, temperatures are given in $\mathrm{keV}, N_{\mathrm{H}}$ in $10^{21} \mathrm{~cm}^{-2}$ and abundances are given with respect to solar.

\begin{tabular}{l|cccc}
\hline \hline Par. & $1(\mathrm{~N})$ & $2(\mathrm{~W})$ & $3(\mathrm{~S})$ & $4(\mathrm{SSE})$ \\
\hline $\mathrm{Y}$ & 4.55 & 2.63 & 1.84 & 1.16 \\
$N_{\mathrm{H}}$ & $2.54 \pm 0.05$ & $2.32 \pm 0.1$ & $2.49 \pm 0.09$ & $2.38 \pm 0.17$ \\
$k T_{\max }$ & $3.18 \pm 0.10$ & $4.15 \pm 0.20$ & $4.2 \pm 0.25$ & $4.9 \pm 0.4$ \\
$k T_{\text {mean }}$ & $2.69 \pm 0.13$ & $3.22 \pm 0.20$ & $3.25 \pm 0.24$ & $3.5 \pm 0.4$ \\
$\alpha$ & $0.22 \pm 0.06$ & $0.41 \pm 0.10$ & $0.41 \pm 0.11$ & $0.6 \pm 0.3$ \\
$\mathrm{Si}$ & $0.60 \pm 0.06$ & $0.70 \pm 0.10$ & $0.59 \pm 0.11$ & $0.50 \pm 0.24$ \\
$\mathrm{~S}$ & $0.46 \pm 0.08$ & $0.43 \pm 0.14$ & $0.17 \pm 0.14$ & $0.31 \pm 0.19$ \\
$\mathrm{Fe}$ & $0.51 \pm 0.02$ & $0.39 \pm 0.03$ & $0.36 \pm 0.03$ & $0.37 \pm 0.04$ \\
\hline$\chi^{2} /$ d.o.f. & $605 / 509$ & $512 / 509$ & $551 / 509$ & $598 / 509$ \\
\hline \multicolumn{5}{|c}{}
\end{tabular}

temperature and iron abundance asymmetry seen in the temperature maps.

\section{Properties of the core on smaller scales}

The high statistics of the data obtained during our deep XMMNewton observation allow us to better study the thermal properties of the blobs/filaments found in the core of 2A 0335+096 with Chandra (Mazzotta et al. 2003). We use a smoothed Chandra ACIS image in the energy band of $0.5-1.5 \mathrm{keV}$ to locate the blobs/filaments. We create three extraction masks: one for the region of bright blobs/filaments; one for the bright region around the blobs, but excluding the blobs (the ambient gas); and one for the bright core but excluding the complex structure (the envelope); see Fig. 8 We use these masks to extract spectra from our EPIC-MOS data. The Full Width Half Maximum (FWHM) of the on-axis Point Spread Function (PSF) of MOS1 and MOS2 at $1.5 \mathrm{keV}$ is $4.3^{\prime \prime}$ and $4.4^{\prime \prime}$ respectively, while the FWHM of the on-axis PSF of EPIC-pn at the same energy is $12.5^{\prime \prime}$. Since the large PSF of EPIC-pn would cause a strong contamination of the spectra extracted from the region of blobs/filaments, we analyze only the data obtained by MOS.

We fit the three spectra simultaneously. The envelope is fitted by a thermal model. The ambient inter-blob gas is fitted by two thermal models, while all the parameters of the second thermal model are coupled to the model with which we fit the envelope. The region of blobs/filaments is fitted with three thermal models, where the parameters of the second and third model are coupled respectively to the model we use to fit the ambient gas, and the envelope. This way we de facto deproject the temperature of the blobs. The only free parameters in the fit are the normalizations of the thermal components, the temperatures, and the iron abundances. The values of the other abundances are fixed to the values determined by fitting the central region with wdem. The redshift is fixed to the redshift of the central cD galaxy. The best fit parameters are shown in Table 9 and the fitted spectra are shown in Fig. 9 The fit shows that the deprojected temperature of the blobs/filaments is $1.14 \pm 0.01$ 


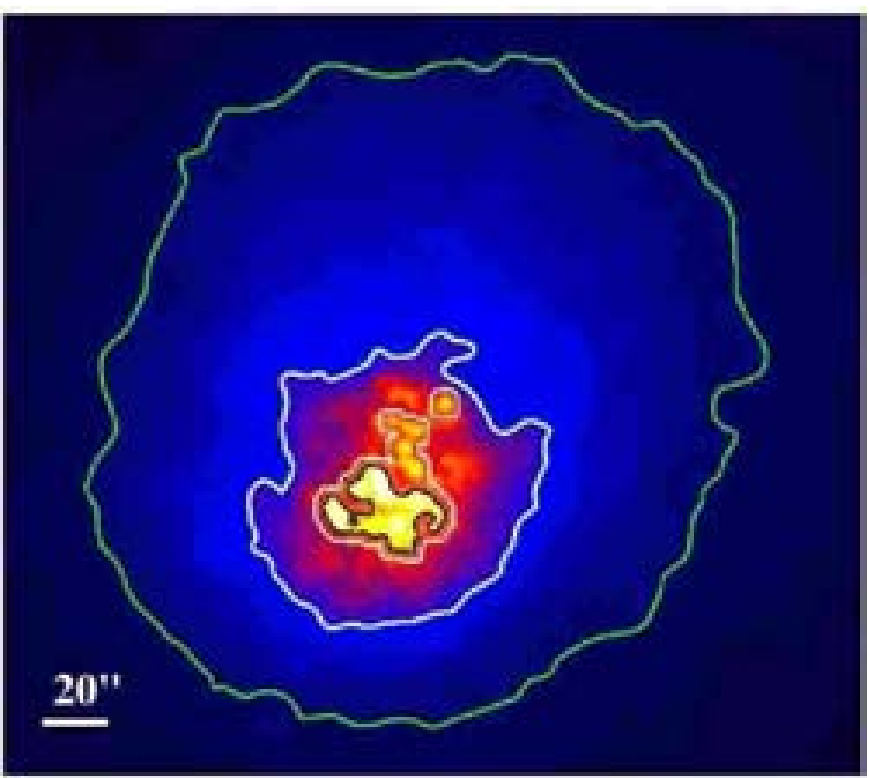

Fig. 8. Smoothed Chandra image of the core of 2A $0335+096$ with overplotted areas used to extract spectra from the data obtained during our deep XMM-Newton observation. The spectra of blobs/filaments, ambient gas and the envelope were extracted respectively from the area indicated by black, white and gray contours.

Table 9. Temperature and iron abundance of the blobs/filaments, the ambient gas around these features and of the envelope of gas surrounding the inner core. The superscript $a$ indicates that the parameters are coupled.

\begin{tabular}{llll}
\hline \hline & blo./fil. & amb. gas & envelope \\
\hline$k T$ & $1.14 \pm 0.01$ & $1.86 \pm 0.01$ & $2.79 \pm 0.01$ \\
$\mathrm{Fe}$ (solar) & $0.63^{a} \pm 0.02$ & $0.63^{a} \pm 0.02$ & $0.61 \pm 0.01$ \\
\hline
\end{tabular}

$\mathrm{keV}$, while the temperature of the ambient gas is $1.86 \pm 0.01$ $\mathrm{keV}$. The iron abundance of the blobs is coupled to the iron abundance of the ambient gas, while the iron abundance of the envelope is fitted separately and is found to be somewhat lower. Coupling the iron abundance of the blobs with that of the ambient gas is necessary to avoid anti-correlation between the two parameters and it has no effect on the determined temperature difference between the two components.

Mazzotta et al. (2003) assumed that the blobs are ellipsoids and selected eight regions. We assume the regions and the luminosities given by Mazzotta et al. (2003) and with our new temperature we calculate with a MEKAL model the density of the blobs. We find a value of $\sim 8.5 \times 10^{-2} \mathrm{~cm}^{-3}$. From our best fit emission measure of the thermal model of the ambient gas we calculate the density of the gas and find a value of $\sim 5.5 \times 10^{-2} \mathrm{~cm}^{-3}$. These density estimates and our new values of the temperatures of these two components therefore indicate, that the cold blobs/filaments and the ambient gas are in thermal pressure equilibrium $\left(n k T \simeq 0.1 \mathrm{keV} \mathrm{cm}^{-3}\right)$.

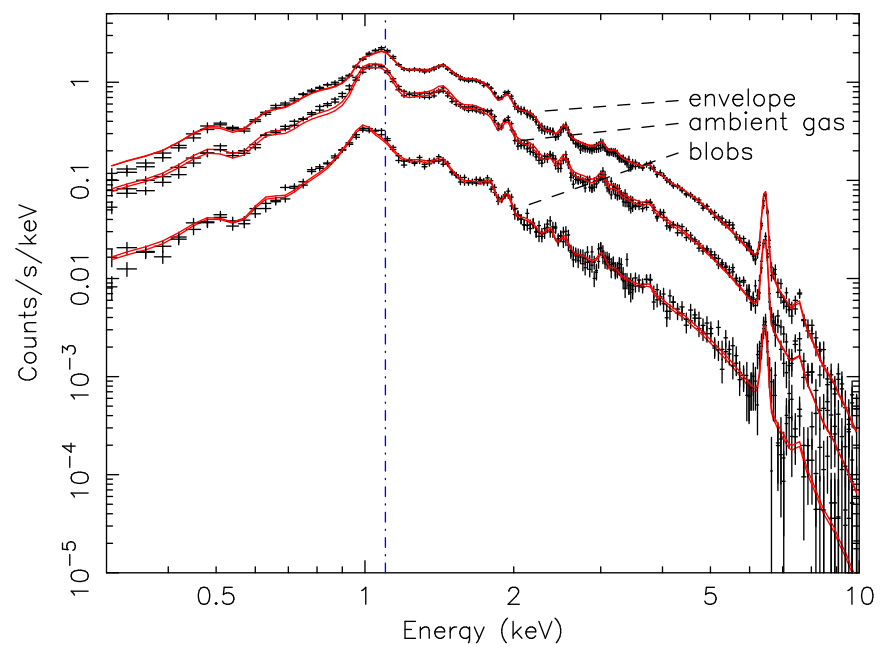

Fig. 9. Spectra of the blobs/filaments, the ambient gas around these features and of the envelope of gas surrounding the inner core. The vertical line helps to see that there is more Fe-L emission at low energies in the spectrum of the blobs.

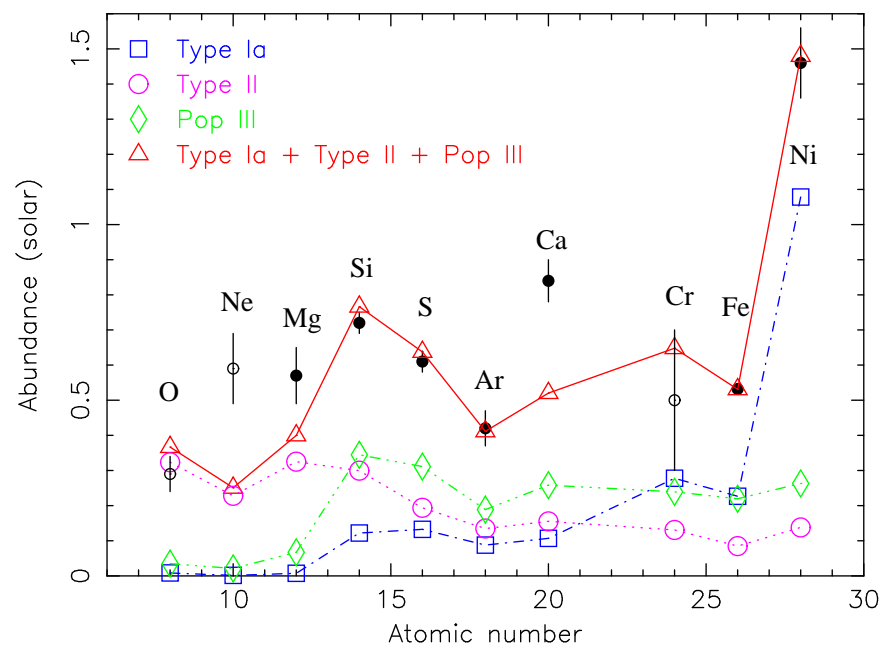

Fig. 10. The elemental abundances derived from a wdem fit of the inner $3^{\prime}$ of $2 \mathrm{~A} 0335+096$, fitted by a linear combination of the yields of SN Ia, SN II and Pop III, for the SN Ia model W7 and Pop III model with a core mass of $130 \mathrm{M}_{\odot}$. The black empty circles indicate elements not used in the fit.

\section{Abundances and enrichment by supernova types la/II and Population III stars}

We investigate the relative contribution of the number of supernova types Ia/II to the total enrichment of the intra-cluster medium (ICM). We also investigate the possibility of putting constraints on the contribution by Population III stars. We try to determine the relative numbers of different supernovae

$\frac{N_{\text {Sx }}}{N_{\text {Ia }}+N_{\text {II }}+N_{\text {popIII }}}$

where $N_{\mathrm{Sx}}$ is either the number of Type Ia supernovae (SNe Ia), Type II supernovae (SNe II) or Popupation III stars (Pop III) contributing to the enrichment of ICM. Since the progenitors of Type II supernovae and Type Ib and Ic (SNe Ib/Ic) are massive 
Table 10. Relative numbers of SN Ia, SN II and Pop III contributing to the enrichment of the intra-cluster medium. We show the results of fits of linear combinations of the yields of SN Ia, SN II and Pop III stars, with two SN Ia yield models, to the abundances of 7 elements shown in Table 2 determined for the core and the outer region of 2A 0335+096. For the core we show the results of fits with two different Pop III star models and the results of fits without Pop III stars.

\begin{tabular}{|c|c|c|c|c|c|c|c|c|c|}
\hline \multirow[t]{2}{*}{ Model } & \multirow[t]{2}{*}{ Type } & \multicolumn{6}{|c|}{$0^{\prime}-3^{\prime}$} & \multicolumn{2}{|c|}{$3^{\prime}-9^{\prime}$} \\
\hline & & $M_{\text {popIII }}=130 \mathrm{M}$ & $\chi^{2} /$ d.o.f. & $M_{\text {popIII }}=65 \mathrm{M}_{\odot}$ & $\chi^{2} /$ d.o.f. & no Pop III & $\chi^{2} /$ d.o.f. & no Pop III & $\chi^{2} /$ d.o.f. \\
\hline \multirow[t]{2}{*}{ W7 } & SN Ia & $0.24 \pm 0.04$ & & $0.18 \pm 0.01$ & & $0.20 \pm 0.01$ & & $0.39 \pm 0.04$ & \\
\hline & $\begin{array}{l}\text { SN II } \\
\text { Pop III }\end{array}$ & $\begin{array}{c}0.76 \pm 0.04 \\
(4 \pm 1) \times 10^{-3}\end{array}$ & $36 / 4$ & $\begin{array}{c}0.85 \pm 0.01 \\
(-2.7 \pm 0.4) \times 10^{-2}\end{array}$ & $60 / 4$ & $\begin{array}{c}0.80 \pm 0.01 \\
-\end{array}$ & $70 / 5$ & $\begin{aligned} 0.61 & \pm 0.04 \\
& -\end{aligned}$ & $44 / 5$ \\
\hline \multirow[t]{2}{*}{ WDD2 } & SN Ia & $0.25 \pm 0.04$ & & $0.27 \pm 0.03$ & & $0.26 \pm 0.01$ & & $0.37 \pm 0.04$ & \\
\hline & $\begin{array}{l}\text { SN II } \\
\text { Pop III }\end{array}$ & $\begin{array}{c}0.75 \pm 0.04 \\
(-2+1) \times 10^{-3}\end{array}$ & $35 / 4$ & $\begin{array}{c}0.72 \pm 0.03 \\
(6+18) \times 10^{-3}\end{array}$ & $43 / 4$ & $\begin{array}{c}0.74 \pm 0.01 \\
-\end{array}$ & $43 / 5$ & $\begin{array}{c}0.63 \pm 0.04 \\
-\end{array}$ & $8 / 5$ \\
\hline
\end{tabular}

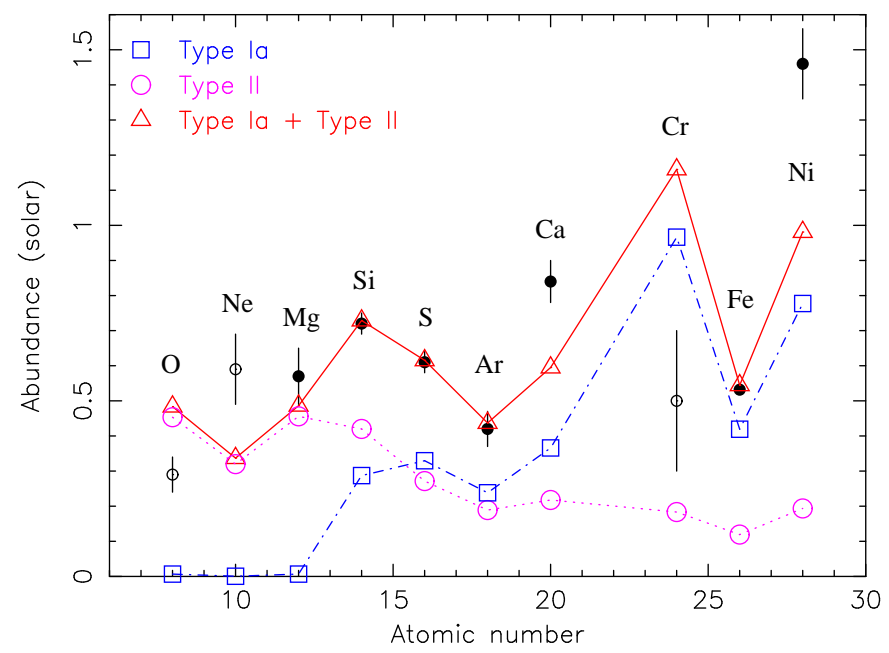

Fig. 11. The elemental abundances derived from a wdem fit of the inner $3^{\prime}$ of $2 \mathrm{~A} 0335+096$, fitted by a linear combination of the yields of SN Ia and SN II. For SN Ia we use the WDD2 model. The black empty circles indicate elements not used in the fit.

stars, we associate the heavy-element yields from SNe II with those from $\mathrm{SNe} \mathrm{Ib} / \mathrm{Ic}$. For nucleosynthesis products of SN II we adopt an average yield of stars on a mass range from $10 \mathrm{M}_{\odot}$ to $50 \mathrm{M}_{\odot}$ calculated by Tsuiimoto et al. (1995) assuming a Salpeter initial mass function. For nucleosynthesis products of SN Ia we adopt values calculated by Iwamoto et al. (1999) and investigate two different models (see Table 10). The W7 model is calculated using a slow deflagration model, while the WDD2 model is calculated using a delayed-detonation model. Note that delayed-detonation models are currently favored over deflagration models by the supernova community, whereas the WDD2 is favored by Iwamoto et al. (1999). The intergalactic medium might also have been significantly enriched by Pop III stars which exploded as pair instability supernovae. We investigate two models for heavy element yields of Pop III stars calculated by Heger \& Wooslev (2002), with a core mass of 130 $\mathrm{M}_{\odot}$ and $65 \mathrm{M}_{\odot}$.

We assume that the observed total number of atoms $N_{i}$ of the element $i$ (determined from its abundance and the emission measure distribution) is a linear combination of the number of atoms $Y_{i}$ produced per individual supernova Type Ia $\left(\mathrm{Y}_{i, \mathrm{Ia}}\right)$, Type II $\left(\mathrm{Y}_{i, \mathrm{II}}\right)$ and Population III star $\left(\mathrm{Y}_{i, \mathrm{III}}\right)$ :

$N_{i}=a \mathrm{Y}_{i, \mathrm{Ia}}+b \mathrm{Y}_{i, \mathrm{II}}+c \mathrm{Y}_{i, \mathrm{III}}$,

where $a, b$ and $c$ are multiplication factors representing the total number of each supernovae that went off in the cluster and enriched the ICM.

First we fit the abundances obtained for the cooling-core region (the inner $3^{\prime}$ ) and determine the best-fit values for $a, b$ and $c$, which we use to estimate the relative numbers of supernovae. We fit the abundance values obtained for the inner $3^{\prime}$ region shown in Table 2 The best fit values of the relative contributions are shown in Table 10 and Fig. 10 In the Fig.10 we also show our best measurement of the chromium abundance and the measured oxygen and neon abundances determined by the RGS and normalized to the iron abundance determined by EPIC (the absolute abundances are not well determined by RGS). Since the low significance makes the value of the chromium abundance uncertain and the abundance values of the oxygen and neon may be affected by systematic uncertainties arising from using different extraction regions for the RGS and EPIC, we do not include them in the fitting. We see that all models are consistent with a scenario where the relative number of Type Ia supernovae contributing to the enrichment of the intra-cluster medium is $20-30 \%$, while the relative number of Type II supernovae is $70-80 \%$.

The $130 \mathrm{M}_{\odot}$ Pop III star model fits show, that their relative number contributing to the enrichment is about two orders of magnitude lower than the number of SN Ia/II. However, they produce according to the models about two orders of magnitude more mass per star than $\mathrm{SN} \mathrm{Ia/II} \mathrm{so} \mathrm{their} \mathrm{contribution} \mathrm{to}$ the enrichment of ICM could still be significant. However, the relative numbers determined for Pop III are model dependent and the high values of the $\chi^{2}$ s do not allow us to confirm their contribution. The $65 \mathrm{M}_{\odot}$ Pop III star model fits give unphysical values for their relative numbers. In Fig. 11] we show our bestfit model of the observed abundances as a linear combination of only SN Ia and SN II. This model gives us the same relative number of SN Ia as the models containing Pop III stars. Also, using the WDD2 SN Ia model, the $\chi^{2}$ does not change with respect to models containing Pop III stars. The fit results show that Pop III stars are not necessary to explain the observed 
abundance patterns, although their contribution to the enrichment of the ICM cannot be excluded. We conclude that this analysis does not allow us to put any constrains on the enrichment by Pop III stars.

In Fig. 10] and [11 we see that the measured abundance of calcium is significantly higher than that predicted by the models. The abundance of nickel is well fitted only using the W7 model of Type Ia supernovae with Pop III stars. Fitting the abundances as a linear combination of SN Ia and SN II, the W7 model predicts more and the WDD2 model predicts less nickel than observed. Mainly the discrepancies between the model and the measured abundances of calcium and nickel are responsible for the large $\chi^{2}$ s. The WDD2 model always predicts significantly more chromium than the upper limit derived from our data-point. The best measured abundances of oxygen and neon are also not well reconstructed by the models. This may be either due to problems in the supernova models or due to the fact that they were determined from a different extraction region with a different instrument. We note that the involved uncertainties and complications when interpreting the observed abundances (see Matteucci \& Chiappini 2005) do not allow us to differentiate between the two SN Ia models.

Since our fits of the core show that we cannot put constrains on the contribution of Pop III stars we fit the "outskirts" $\left(3^{\prime}-9^{\prime}\right)$ with models containing only SN Ia and SN II. The fits show a higher contribution of SN Ia in the outer region than in the cooling core region, however the large $\chi^{2}$ values and the involved systematic uncertainties do not allow us to make a firm statement.

\section{Discussion}

We have used three different thermal models in our analysis of 2A 0335+096 and find that the multi-temperature wdem model fits the data best. We note that a good description of the temperature structure is very important to obtain correct values for elemental abundances. Fitting the EPIC and RGS data we determine the radial temperature and abundance profiles for several elements. The elemental abundances have a peak in the core of the cluster and show a gradient across the whole cluster. The abundance structure of the cluster is consistent with a scenario where the relative number of SN Ia is $20-30 \%$ of the total number of supernovae enriching the ICM. Contrary to the findings of Baumgartner et al. (2005), the Population III stars are not necessary to explain the observed abundance patterns. However, their contribution to the enrichment of the ICM cannot be excluded. Extracting temperature and iron abundance maps we find an asymmetry across the cluster. We find that the deprojected temperature of the blobs/filaments in the core of the cluster is lower than the temperature of the ambient gas and the blobs appear to be in thermal pressure equilibrium with the ambient gas. Below, we discuss the implications of these results.

\subsection{Intrinsic temperature structure}

Our results show a presence of plasma emitting at a range of different temperatures within every extraction bin. In the core of the cluster, this may be caused in part by projection effects and by the strong temperature gradient. The multi-temperature structure we find around the central bin may also be caused in part by the large scale temperature asymmetry of the cluster, so in one extraction annulus we extract the spectrum of both the colder and of the warmer gas from North and South of the cluster center respectively. However, the non-isothermality detected by fitting the deprojected spectra cannot be caused only by the width of our extraction annuli in combination with the temperature asymmetry. The temperature gradients across our annuli and the temperature differences between North and South are not large enough for that. The results can be interpreted as intrinsic multi-temperature structure at each position, which is in agreement with the results of Kaastra et al. (2004). Furthermore, these results show that fitting the spectrum of the intra-cluster gas with a single-temperature thermal model is in general not sufficient and introduces systematic errors especially in the determination of elemental abundances.

\subsubsection{Spatial temperature distribution}

The temperature distribution has a strong gradient in the core. In the inner $3^{\prime}$ the deprojected temperature drops by a factor of $\sim 1.8$ toward the center. In the outer part $\left(3^{\prime}-9^{\prime}\right)$ the radial temperature distribution is flat. We do not see a temperature drop toward the outskirts. We find an asymmetry in the temperature distribution and iron abundance distribution. The temperature maps show the asymmetry in temperature distribution in the direction of the elongated surface brightness morphology, roughly in the South-Southeast North-Northwest direction. The temperature drops faster toward the core from the South than from the North. The temperature change is the strongest over the cold front South of the core identified by Mazzotta et al. (2003) in Chandra data. The cold fronts are interpreted as boundaries of a dense, cooler gas moving through the hotter, more rarefied ambient gas (Markevitch et al. 2000). From the pressure jump across the cold front Mazzotta et al. (2003) conclude that the dense central gas core moves from North to South with a Mach number of $M \simeq 0.75 \pm 0.2$. The elongated, comet-like shape of the cold central core shown in our temperature maps supports this picture. The elongated shape of the cold gas core may hint that we see a surviving core of a merged subcluster moving through and being stripped by a less dense surrounding gas. The possible merger scenario is discussed in subsection 9.3

\subsubsection{X-ray blobs/filaments}

Mazzotta et al. (2003) found that the deprojected temperature of the blobs is consistent with that of the less dense ambient gas, so these gas phases do not appear to be in thermal pressure equilibrium. Therefore they propose a possibility of a significant unseen non-thermal pressure component in the inter-blob gas, possibly arising from the activity of a central active galactic nucleus (AGN). They also discuss two models to explain the origin of the blobs: hydrodynamic instabilities caused by 
the motion of the cool core and "bubbling" of the core caused by multiple outbursts of the central AGN.

However, the temperature measurements of Mazzotta et al. (2003) have large uncertainties due to the low statistics of the Chandra data. The superior spectral quality of our XMMNewton data allows us to measure the temperature of the blobs much more accurately. In contrast to the previous findings we find that the deprojected temperature of the region where they identified the blobs/filaments is colder by a factor of 1.6 than the temperature of the ambient gas and the blobs appear to be in thermal pressure equilibrium with the ambient gas. In the light of this new result a non-thermal pressure component in the inter-blob gas is not necessary to explain the observed blobs.

\subsection{Abundance distribution}

The high statistics of the data allow us to determine the abundances of the most abundant metals. For the first time we put constraints on the abundance of chromium, and upper limits on abundances of titanium, manganese and cobalt. The abundance distribution has a peak in the core of the cluster. The radial profiles show an abundance gradient toward the core across the whole analyzed region of the cluster. The abundance gradient is not restricted to the cooling-flow region. We observe centrally peaked abundance distributions for both SN Ia products (iron, nickel) and elements contributed mainly by SN II (magnesium, silicon). Contrary to our results from 2A 0335+096, cooling core clusters (e.g. see the sample of Tamura et al. (2004)) in general show a centraly peaked distribution of SN Ia products with respect to SN II products. The different abundance distribution in 2A 0335+096 might point toward a different enrichment history. In Sérsic 159-03 the widths of the RGS lines also indicate that the iron abundance is much more centrally peaked than oxygen (de Plaa et al. 2006). Contrary to Sérsic 159-03, the RGS data in 2A 0335+096 indicate that the spatial distributions of oxygen and iron might be very similar. However, we have to be careful with interpreting the RGS results, because the strong temperature drop in the center of this cluster might cause that the RGS spectrum is dominated by the line emission from the cool core and the line profiles of iron and oxygen follow the temperature structure more than the abundance distribution of the elements.

We estimate that the total mass of iron within the radius of $126 \mathrm{kpc}$ is $\sim 2.1 \times 10^{9} \mathrm{M}_{\odot}$. If all iron originates from SN Ia, we need $\sim 2.8 \times 10^{9} \mathrm{SN}$ Ia explosions to explain the observed amount of iron. Assuming $1.1 \times 10^{10}$ years and 50 galaxies contributing iron via galactic winds and gas stripping we get an average SN Ia rate of one explosion per 200 years per galaxy.

We show that the determined abundance values are sensitive to the applied temperature model. Especially singletemperature thermal models can introduce a bias in the abundance determination, resulting in a detection of off-center abundance peaks reported for example by Sanders \& Fabian (2002) and Johnstone et al. (2002).

The abundance maps show an asymmetry in the iron abundance distribution, with a higher abundance to the North of the cluster core. The abundance asymmetry further highlights and supports the picture of great dynamical complexity.

\subsection{Possible merger scenario}

We observe an asymmetry of the temperature and iron abundance distribution across the cluster, along the surface brightness elongation, which points toward the possibility of an ongoing merger in 2A 0335+096. Mazzotta et al. (2003) note that the orientation of the cold front and the asymmetry of the temperature distribution suggest that the cool core is moving along a projected direction that goes from North to South. However, it is not known whether the motion is due to a merger, or due to gas sloshing induced by an off-axis merger or by a flyby of another cluster. Mazzotta et al. (2003) also reason that if the cool core is indeed a merging subcluster, then in projection the position of the subcluster is close to the cluster center. However, the impact parameter cannot be measured hence the subcluster may be passing through the cluster at any distance along the line of sight. Our detection of an asymmetry in the abundance distribution across the cluster, with a higher metallicity North of the core with respect to the South, supports the merger scenario, in which the subcluster had a higher metallicity. The most important enrichment processes, like ram pressure stripping of cluster galaxies and galactic winds, are not expected to produce the observed asymmetry in the spatial distribution of the iron abundance. In the dense cores of clusters of galaxies the internal kinematics of the ICM is expected to quickly smooth out any existing inhomogeneities in the abundance distribution.

In Fig. 12 we show an archival Hubble Space Telescope (HST) image of the core of the cluster with overplotted EPICMOS X-ray isophots. The HST image shows the central cD galaxy and a companion galaxy to the North-West (the bright source in the lower left is a foreground star). We see, that the $\mathrm{X}$-ray brightness peak is offset to the South from the central $\mathrm{cD}$ galaxy, which also shows that the core is not in a relaxed state. This was already shown by Mazzotta et al. (2003) who found that the centroid positions of the best fit double $\beta$-model to the cluster surface brightness do not coincide with the position of the central cD galaxy, but are offset to the South-East and North-West. It is interesting to note, that the alignment of the central $\mathrm{cD}$ galaxy and the alignment of the central galaxy with the projected companion nucleus are following the same direction as the possible merger axis inferred from the X-rays. Dubinski (1998) has shown that the long axis of a brightest cluster galaxy always aligns with the primordial filament along which the cluster collapsed and with the long axis of the cluster galaxy distribution. If 2A 0335+096 formed along a filament aligned as the long axis of the central cD galaxy, we might be seeing the traces of the latest phase of the formation of the cluster, in which a subcluster is falling in along the same primordial filament.

Although there is growing evidence for the merger scenario, with the available data we still cannot rule out an alternative scenario of gas sloshing. Radial velocity measurements of 


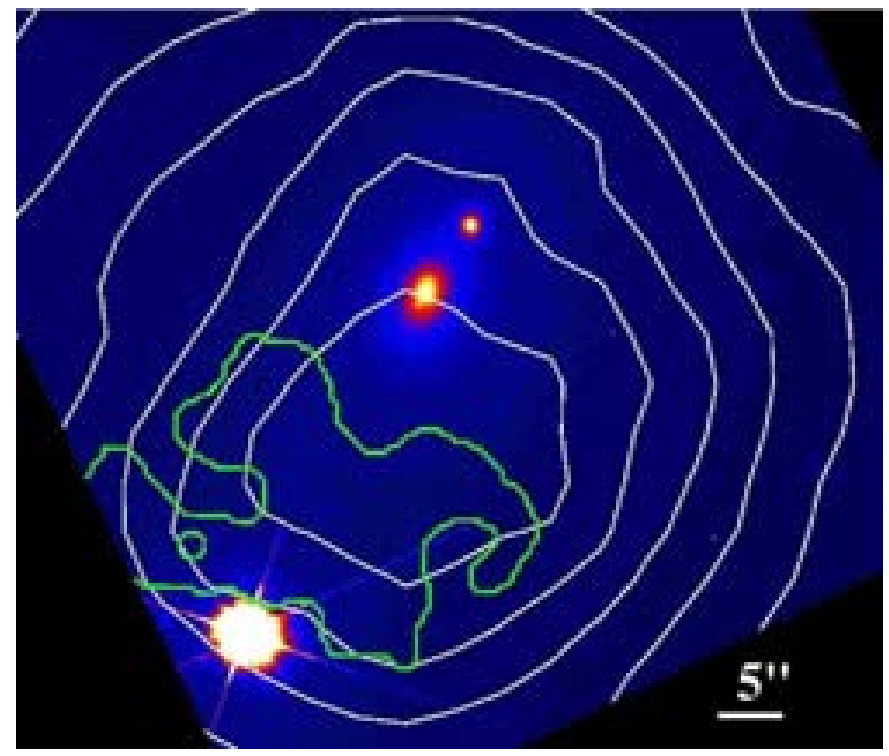

Fig. 12. An image of the core of the cluster obtained by the Hubble Space Telescope with overplotted X-ray isophots. North is up, West is right. The X-ray isophots are off-set from the central $\mathrm{cD}$ galaxy. The gray contour indicates the location of the cold blobs/filaments detected in X-rays.

the individual galaxies in 2A $0335+096$ are necessary to provide the decisive evidence.

\subsection{Relative enrichment by supernova types la/ll and Population III stars}

All our fits show that the contribution of $\mathrm{SN}$ Ia is $20-$ $30 \%$ of the total number of supernovae enriching the ICM. Tsuiimoto et al. (1995) found that the ratio of the total numbers (integrated over time) of supernovae Type Ia to Type II that best reproduces the Galactic abundances is $N_{\text {Ia }} / N_{\text {II }}=0.15$, while the ratio determined for the Magellanic Clouds is $0.2-0.3$, which correspond to relative numbers of $N_{\text {Ia }} / N_{\text {Ia }+ \text { II }}=0.13$ and $0.17-0.23$ in the Galaxy and in the Magellanic clouds respectively. Supernova types Ia/II produce heavy-elements on different time scales during the chemical evolution of galaxies, with SN II causing the enrichment usually in the early phases of galactic evolution and $\mathrm{SNe}$ Ia on a much longer time-scale, in the later phases of galactic evolution. The current relative frequencies of SNe Ia and II also depend on types of galaxies. According to the Lick Observatory Supernova Search (LOSS) about $42 \%$ of the supernovae observed in galaxies within a redshift of $z=0.03$ are $\mathrm{SN}$ Ia, while SNe II are rare in early-type galaxies and $\mathrm{SNe}$ Ia occur in all types of galaxies (van den Bergh et al. 2005). The relative contribution to the enrichment of ICM by SN Ia in the cluster of galaxies 2A $0335+096$ is between the Galactic value and the value of the current relative frequencies determined by LOSS (van den Bergh et al. 2005). The value of $N_{\text {Ia }} / N_{\text {Ia+II }}=20-30 \%$ is consistent with a picture of an early enrichment by $\mathrm{SNe}$ II material from star-burst galaxies and later contribution by Type Ia supernovae material.
However, the scenario proposed by Tamura et al. (2001a) and Finoguenov et al. (2001), where an early and well mixed SN II enrichment is followed by SN Ia enrichment in the central galaxy, produces over the Hubble time a centrally peaked distribution of SN Ia products relative to SN II products. As discussed in 9.2 we observe a centrally peaked abundance distribution of both SN Ia and SN II products. Moreover, contrary to the expectations, comparing the relative enrichment in the inner and outer regions of the cluster the $\mathrm{Mg} / \mathrm{Fe}, \mathrm{Si} / \mathrm{Fe}$ and $\mathrm{S} / \mathrm{Fe}$ ratios point toward a higher contribution of $\mathrm{SN}$ Ia in the outer parts compared to the cool core. Such a difference in the enrichment history between the core and the outer parts would strongly support the scenario according to which the cool core is the surviving ICM of a merged subcluster (see 9.3). However, the involved uncertainties in the determination of the relative enrichment are high.

As discussed by Matteucci \& Chiappini (2005) the interpretation of the observed abundance ratios is not easy. The observed abundance ratios strongly depend on the star-formation history in cluster galaxies. Part of the supernova products might still be locked in the stars or in the potential wells of cluster galaxies. Therefore, the relative number of supernovae contributing to the enrichment of the ICM, which we infer from the abundance ratios, might be somewhat different from the relative number of supernovae which exploded in the cluster over its life-time.

The results also show that it is not necessary to include the Pop III stars in our models to explain the observed abundance patterns. Our magnesium, silicon, sulfur, argon and iron abundances can be reconstructed with a linear combination of yields of SN Ia and II for all tested SN Ia yield models. In the cluster 2A $0335+096$ we cannot confirm the results of Baumgartner et al. (2005) claiming, that especially in the hotter clusters, Pop III stars are necessary to explain the observed silicon, sulfur, calcium and argon abundances. However, to test the results of Baumgartner et al. (2005) an analysis of a large cluster sample including hotter clusters will be necessary.

All fitted models give a significantly lower calcium abundance than we observe. This points toward uncertainties in the models of SN II yields, e.g. different explosion energies in the models can result in a difference of an order of magnitude in calcium production (Woosley \& Weaver 1995).

\section{Conclusions}

We have analyzed spatially resolved and high resolution spectra of the cluster of galaxies 2A 0335+096 obtained during a deep XMM-Newton observation. We found that:

- We unambiguously detect multi-temperature structure in the core of 2A 0335+096: for the determination of elemental abundances, a multi-temperature model is mandatory. The wdem model is a good description of the temperature structure of the gas in 2A $0335+096$.

- The blobs/filaments found in the core of 2A 0335+096 are significantly colder than the ambient gas and they appear to be in pressure equilibrium with their environment. 
- We detect a strong central peak in the abundance distributions of both SN Ia and SN II products.

- The relative number of SN Ia contributing to the enrichment of the intra-cluster medium in the central $130 \mathrm{kpc}$ of 2A $0335+096$ is $\sim 25 \%$, while the relative number of SN II is $\sim 75 \%$. Comparison of the observed abundances to the supernova yields does not allow us to put any constrains on the contribution of Pop III stars to the enrichment of the ICM. We observe significantly higher calcium abundance than predicted by supernova models.

- The detected asymmetry in the temperature and iron abundance distribution further supports the merger scenario, in which the subcluster had a higher metallicity.

Acknowledgements. This work is based on observations obtained with XMM-Newton, an ESA science mission with instruments and contributions directly funded by ESA member states and the USA (NASA). The Netherlands Institute for Space Research (SRON) is supported financially by NWO, the Netherlands Organization for Scientific Research.

\section{References}

Allende Prieto, C., Lambert, D. L., \& Asplund, M. 2001, ApJ, 556, L63

Anders, E. \& Grevesse, N. 1989, Geochim. Cosmochim. Acta, 53, 197

Baumgartner, W. H., Loewenstein, M., Horner, D. J., \& Mushotzky, R. F. 2005, ApJ, 620, 680

Bonamente, M., Lieu, R., Mittaz, J. P. D., Kaastra, J. S., \& Nevalainen, J. 2005, ApJ, 629, 192

Buote, D. A. 2000, MNRAS, 311, 176

Cooke, B. A., Ricketts, M. J., Maccacaro, T., et al. 1978, MNRAS, 182, 489

Davis, J. E. 2001, ApJ, 548, 1010

De Grandi, S. \& Molendi, S. 2001, ApJ, 551, 153

De Grandi, S. \& Molendi, S. 2002, ApJ, 567, 163

De Luca, A. \& Molendi, S. 2004, A\&A, 419, 837

de Plaa, J., Kaastra, J. S., Méndez, M., et al. 2005, ASR, in press.

de Plaa, J., Kaastra, J. S., Tamura, T., et al. 2004, A\&A, 423, 49

de Plaa, J., Werner, N., Bykov, A., et al. 2006, A\&A, submitted den Herder, J. W., Brinkman, A. C., Kahn, S. M., et al. 2001, A\&A, 365, L7

Dickey, J. M. \& Lockman, F. J. 1990, ARA\&A, 28, 215

Dubinski, J. 1998, ApJ, 502, 141

Edge, A. C. 2001, MNRAS, 328, 762

Ettori, S., De Grandi, S., \& Molendi, S. 2002, A\&A, 391, 841

Fabian, A. C. 1994, ARA\&A, 32, 277

Finoguenov, A., Arnaud, M., \& David, L. P. 2001, ApJ, 555, 191

Grevesse, N. \& Sauval, A. J. 1998, Space Science Reviews, 85, 161

Heger, A. \& Woosley, S. E. 2002, ApJ, 567, 532

Irwin, J. A. \& Sarazin, C. L. 1995, ApJ, 455, 497

Iwamoto, K., Brachwitz, F., Nomoto, K., et al. 1999, ApJS, 125,439

Jansen, F., Lumb, D., Altieri, B., et al. 2001, A\&A, 365, L1
Johnstone, R. M., Allen, S. W., Fabian, A. C., \& Sanders, J. S. 2002, MNRAS, 336, 299

Kaastra, J. S., Ferrigno, C., Tamura, T., et al. 2001, A\&A, 365, L99

Kaastra, J. S., Lieu, R., Tamura, T., Paerels, F. B. S., \& den Herder, J. W. 2003, A\&A, 397, 445

Kaastra, J. S., Mewe, R., \& Nieuwenhuijzen, H. 1996, in UV and X-ray Spectroscopy of Astrophysical and Laboratory Plasmas p.411, K. Yamashita and T. Watanabe. Tokyo : Universal Academy Press

Kaastra, J. S., Tamura, T., Peterson, J. R., et al. 2004, A\&A, 413, 415

Kikuchi, K., Furusho, T., Ezawa, H., et al. 1999, PASJ, 51, 301

Kuntz, K. D. \& Snowden, S. L. 2000, ApJ, 543, 195

Lodders, K. 2003, ApJ, 591, 1220

Lumb, D. H., Warwick, R. S., Page, M., \& De Luca, A. 2002, A\&A, 389, 93

Markevitch, M., Ponman, T. J., Nulsen, P. E. J., et al. 2000, ApJ, 541, 542

Matteucci, F. \& Chiappini, C. 2005, Publications of the Astronomical Society of Australia, 22, 49

Mazzotta, P., Edge, A. C., \& Markevitch, M. 2003, ApJ, 596, 190

Molendi, S. \& Gastaldello, F. 2001, A\&A, 375, L14

Moretti, A., Campana, S., Lazzati, D., \& Tagliaferri, G. 2003, ApJ, 588, 696

Peterson, J. R., Kahn, S. M., Paerels, F. B. S., et al. 2003, ApJ, 590, 207

Peterson, J. R., Paerels, F. B. S., Kaastra, J. S., et al. 2001, A\&A, 365, L104

Read, A. M. \& Ponman, T. J. 2003, A\&A, 409, 395

Romanishin, W. \& Hintzen, P. 1988, ApJ, 324, L17

Sanders, J. S. \& Fabian, A. C. 2002, MNRAS, 331, 273

Sarazin, C. L., Baum, S. A., \& O’Dea, C. P. 1995, ApJ, 451, 125

Sarazin, C. L., O’Connell, R. W., \& McNamara, B. R. 1992, ApJ, 397, L31

Schwartz, D. A., Schwarz, J., \& Tucker, W. 1980, ApJ, 238, L59

Singh, K. P., Westergaard, N. J., \& Schnopper, H. W. 1986, ApJ, 308, L51

Singh, K. P., Westergaard, N. J., \& Schnopper, H. W. 1988, ApJ, 331, 672

Strüder, L., Briel, U., Dennerl, K., et al. 2001, A\&A, 365, L18

Tamura, T., Bleeker, J. A. M., Kaastra, J. S., Ferrigno, C., \& Molendi, S. 2001a, A\&A, 379, 107

Tamura, T., Kaastra, J. S., den Herder, J. W. A., Bleeker, J. A. M., \& Peterson, J. R. 2004, A\&A, 420, 135

Tamura, T., Kaastra, J. S., Peterson, J. R., et al. 2001b, A\&A, 365, L87

Tsujimoto, T., Nomoto, K., Yoshii, Y., et al. 1995, MNRAS, 277,945

Turner, M. J. L., Abbey, A., Arnaud, M., et al. 2001, A\&A, 365, L27

van den Bergh, S., Li, W., \& Filippenko, A. V. 2005, PASP, in press.

Weisskopf, M. C., O’Dell, S. L., Paerels, F., et al. 2004, ApJ, 601,1050 


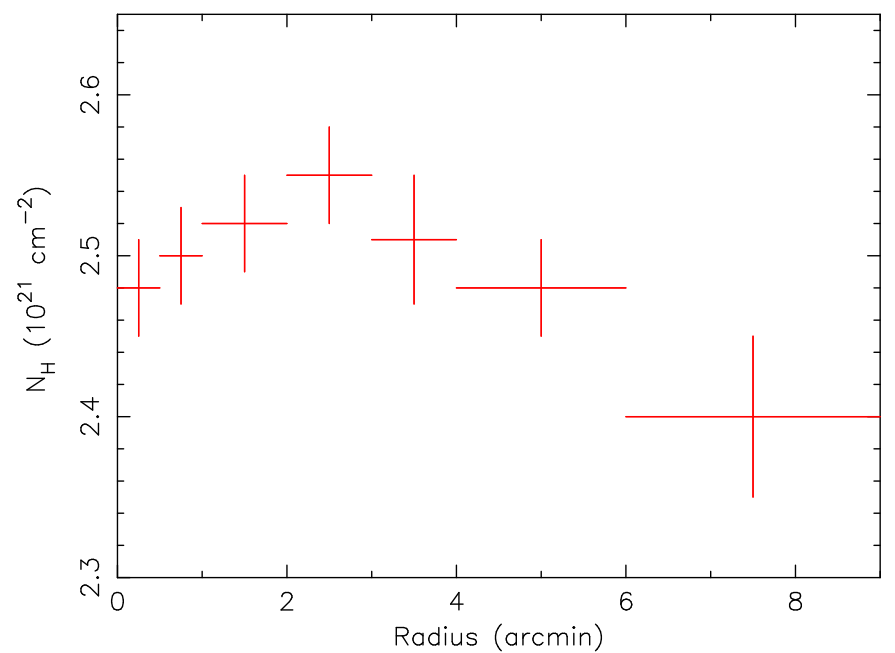

Fig. A.1. The radial hydrogen column density profile.

White, D. A., Fabian, A. C., Johnstone, R. M., Mushotzky, R. F., \& Arnaud, K. A. 1991, MNRAS, 252, 72

Woosley, S. E. \& Weaver, T. A. 1995, ApJS, 101, 181

Zwicky, F., Karpowicz, M., \& Kowal, C. T. 1965, in Catalog of Galaxies and Clusters of Galaxies Vol. 5 (Pasadena: California Institute of Technology)

\section{Appendix A: The influence of the background subtraction on radial profiles}

We compare the radial temperature and abundance profiles obtained using the combined background event lists of Read \& Ponman (2003) with those determined using the background model described in subsection 2.1.1 We compare the values obtained using the wdem model, which was shown to describe the spectrum in the cool cores of clusters well (see Sect. 3). We derive a radial hydrogen column density profile, maximum temperature profile, $\alpha$ profile and abundance profiles of several elements: magnesium, silicon, sulfur, argon, calcium, iron and nickel.

Fitting the data using the background event files of Read \& Ponman (2003) we find an average hydrogen column density of $(2.49 \pm 0.03) \times 10^{21} \mathrm{~cm}^{-2}$, which is significantly higher than the Galactic value of $1.80 \times 10^{21} \mathrm{~cm}^{-2}$ determined from the $21 \mathrm{~cm}$ radio emission (Dickey \& Lockman 1990). This high absorption column density was found before in ROSAT PSPC spectra by Irwin \& Sarazin (1995) and in a previous, shorter XMM-Newton observation by Kaastra et al. (2003). The additional absorption might be caused by Galactic dust clouds or molecular clouds. The measured radial absorption distribution has a slight peak between $2^{\prime}$ and $3^{\prime}$, but the errobars are large and it is still consistent with a flat distribution (see Fig. A.1). Therefore, when fitting the data with our background model, we fix the absorption to $2.5 \times 10^{21} \mathrm{~cm}^{-2}$.

The left top panel in Fig. A.2 shows that the radial temperature profiles derived with the background event files of Read \& Ponman (2003) and with our background model are consistent with each other up to $6^{\prime}$ from the core of the cluster. The best fit abundance values are, however, consistent only up to $4^{\prime}$ from the core and in the outer parts, where the background starts to play a more important role the differences are large. This is shown in Fig. A.2 and Table A.1 We see that using the new background modeling the iron, silicon and magnesium abundances between $4^{\prime}-9^{\prime}$ from the core are lower than those derived using the standard background event files. The large magnesium abundances at the outer radii obtained using the standard background event files are due to the incorrect subtraction of the instrumental aluminum lines. Abundances of other elements are consistent within the errorbars. 

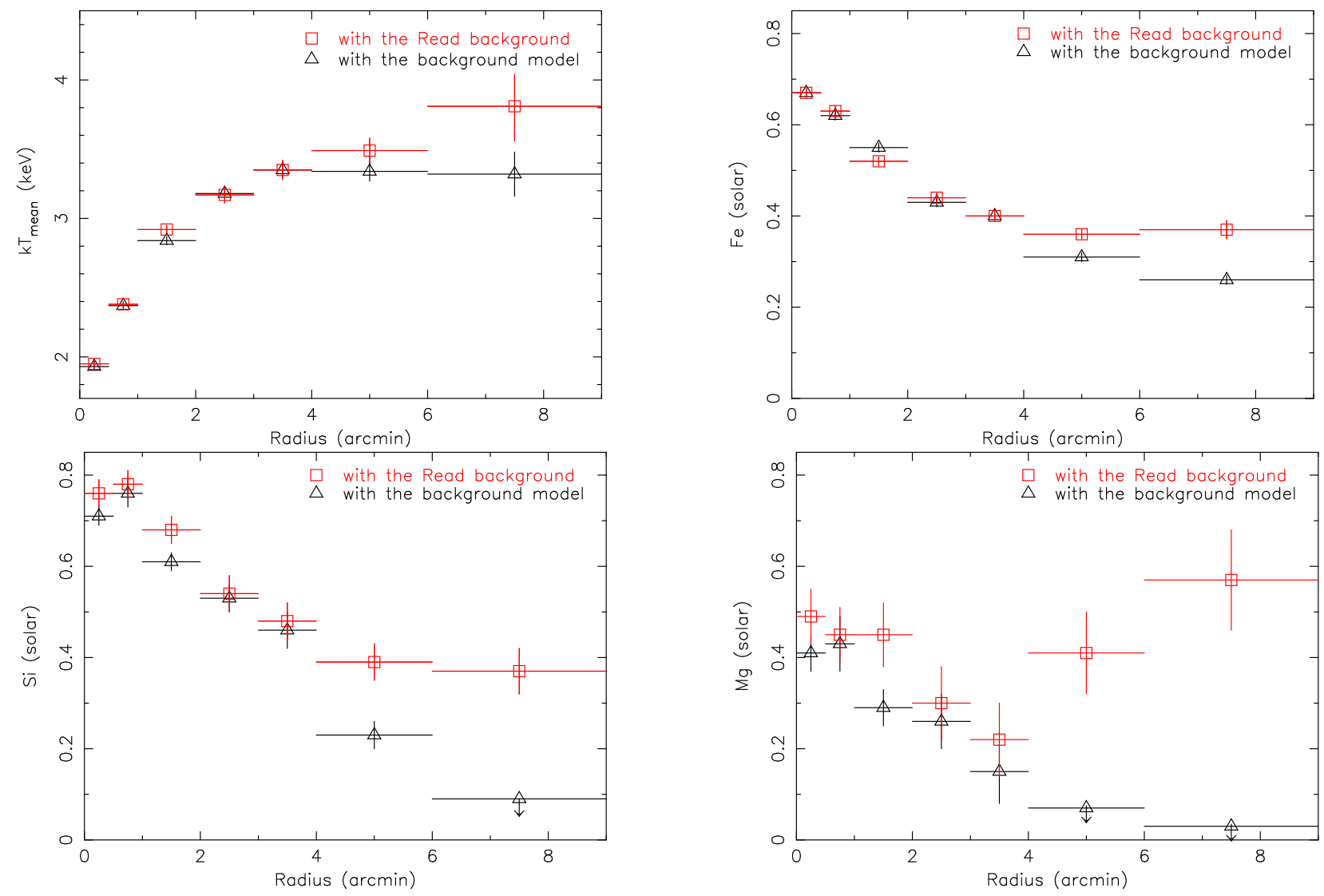

Fig. A.2. Comparison of fit results obtained by fitting the wdem model to EPIC spectra using the combined background event files of Read \& Ponman (2003) and using our new background subtraction method. 
Table A.1. Fit results obtained by fitting the wdem model to EPIC spectra using $(R)$ the combined background event lists of Read \& Ponman (2003) and using $(M)$ the new background subtraction method. Abundances are given with respect to solar.

\begin{tabular}{|c|c|c|c|c|c|c|c|c|}
\hline Parameter & backg. & $0-0.5^{\prime}$ & $0.5-1.0^{\prime}$ & $1.0-2.0^{\prime}$ & $2.0-3.0^{\prime}$ & $3.0-4.0^{\prime}$ & $4.0-6.0^{\prime}$ & $6.0-9.0^{\prime}$ \\
\hline$N_{\mathrm{H}}\left(10^{21} \mathrm{~cm}^{-2}\right)$ & $\mathrm{R}$ & $2.48 \pm 0.03$ & $2.50 \pm 0.03$ & $2.52 \pm 0.03$ & $2.55 \pm 0.03$ & $2.51 \pm 0.04$ & $2.48 \pm 0.03$ & $2.40 \pm 0.05$ \\
\hline$k T_{\max }(\mathrm{keV})$ & $\mathrm{R}$ & $2.66 \pm 0.02$ & $3.16 \pm 0.02$ & $3.98 \pm 0.04$ & $4.40 \pm 0.06$ & $4.73 \pm 0.07$ & $5.04 \pm 0.10$ & $6.28 \pm 0.23$ \\
\hline$k T_{\max }(\mathrm{keV})$ & M & $2.64 \pm 0.02$ & $3.14 \pm 0.02$ & $3.95 \pm 0.03$ & $4.39 \pm 0.04$ & $4.81 \pm 0.05$ & $4.97 \pm 0.05$ & $5.84 \pm 0.14$ \\
\hline$k T_{\text {mean }}(\mathrm{keV})$ & $\bar{R}$ & $1.95 \pm 0.02$ & $2.38 \pm 0.02$ & $2.92 \pm 0.03$ & $3.17 \pm 0.06$ & $3.35 \pm 0.07$ & $3.49 \pm 0.09$ & $3.81 \pm 0.25$ \\
\hline$k T_{\text {mean }}(\mathrm{keV})$ & $\mathrm{M}$ & $1.93 \pm 0.02$ & $2.37 \pm 0.02$ & $2.84 \pm 0.03$ & $3.18 \pm 0.04$ & $3.35 \pm 0.04$ & $3.34 \pm 0.07$ & $3.32 \pm 0.16$ \\
\hline$\alpha$ & $\mathrm{R}$ & $0.57 \pm 0.01$ & $0.49 \pm 0.02$ & $0.57 \pm 0.02$ & $0.64 \pm 0.05$ & $0.71 \pm 0.06$ & $0.83 \pm 0.07$ & $2.4_{-0.5}^{+0.8}$ \\
\hline \multirow[t]{2}{*}{$\alpha$} & M & $0.58 \pm 0.01$ & $0.48 \pm 0.02$ & $0.65 \pm 0.02$ & $0.62 \pm 0.04$ & $0.79 \pm 0.04$ & $1.01_{-0.02}^{+0.23}$ & $2.37_{-3}^{+13}$ \\
\hline & M & $0.95 \pm 0.07$ & $0.79 \pm 0.10$ & $0.16 \pm 0.06$ & $0.1 \pm 0.08$ & $<0.03$ & $<0.01$ & $<0.01$ \\
\hline \multirow[t]{2}{*}{$\mathrm{Mg}$} & $\mathrm{R}$ & $0.49 \pm 0.06$ & $0.45 \pm 0.06$ & $0.45 \pm 0.07$ & $0.30 \pm 0.08$ & $0.22 \pm 0.08$ & $0.41 \pm 0.09$ & $0.57 \pm 0.11$ \\
\hline & M & $0.41 \pm 0.04$ & $0.43 \pm 0.06$ & $0.29 \pm 0.04$ & $0.26 \pm 0.06$ & $0.15 \pm 0.07$ & $<0.07$ & $<0.03$ \\
\hline \multirow[t]{2}{*}{$\overline{\mathrm{Si}}$} & $\overline{\mathrm{R}}$ & $0.76 \pm 0.03$ & $0.78 \pm 0.03$ & $0.68 \pm 0.03$ & $0.54 \pm 0.04$ & $0.48 \pm 0.04$ & $0.39 \pm 0.04$ & $0.37 \pm 0.05$ \\
\hline & M & $0.71 \pm 0.02$ & $0.76 \pm 0.03$ & $0.61 \pm 0.02$ & $0.53 \pm 0.03$ & $0.46 \pm 0.04$ & $0.23 \pm 0.03$ & $<0.09$ \\
\hline \multirow[t]{2}{*}{$\bar{S}$} & $\overline{\mathrm{R}}$ & $0.70 \pm 0.03$ & $0.68 \pm 0.03$ & $0.53 \pm 0.03$ & $0.40 \pm 0.04$ & $0.35 \pm 0.05$ & $0.20 \pm 0.05$ & $<0.15$ \\
\hline & M & $0.67 \pm 0.03$ & $0.65 \pm 0.04$ & $0.51 \pm 0.03$ & $0.38 \pm 0.05$ & $0.35 \pm 0.06$ & $0.12 \pm 0.07$ & $<0.10$ \\
\hline \multirow[t]{2}{*}{$\mathrm{Ar}$} & $\overline{\mathrm{R}}$ & $0.66 \pm 0.06$ & $0.55 \pm 0.07$ & $0.35 \pm 0.08$ & $0.20 \pm 0.10$ & $0.32 \pm 0.12$ & $<0.21$ & $<0.04$ \\
\hline & M & $0.69 \pm 0.06$ & $0.50 \pm 0.09$ & $0.35 \pm 0.08$ & $0.19 \pm 0.12$ & $0.24 \pm 0.14$ & $<0.18$ & $<0.07$ \\
\hline \multirow[t]{2}{*}{$\mathrm{Ca}$} & $\overline{\mathrm{R}}$ & $0.74 \pm 0.10$ & $0.97 \pm 0.11$ & $0.98 \pm 0.13$ & $0.79 \pm 0.17$ & $0.78 \pm 0.18$ & $0.53 \pm 0.15$ & $<0.25$ \\
\hline & M & $0.74 \pm 0.09$ & $0.98 \pm 0.11$ & $1.02 \pm 0.10$ & $0.77 \pm 0.15$ & $0.74 \pm 0.17$ & $0.66 \pm 0.17$ & $0.70 \pm 0.23$ \\
\hline \multirow[t]{2}{*}{$\mathrm{Fe}$} & $\mathrm{R}$ & $0.67 \pm 0.01$ & $0.63 \pm 0.01$ & $0.52 \pm 0.01$ & $0.44 \pm 0.01$ & $0.40 \pm 0.01$ & $0.36 \pm 0.01$ & $0.37 \pm 0.02$ \\
\hline & M & $0.67 \pm 0.01$ & $0.62 \pm 0.01$ & $0.55 \pm 0.01$ & $0.43 \pm 0.01$ & $0.40 \pm 0.01$ & $0.31 \pm 0.01$ & $0.26 \pm 0.01$ \\
\hline \multirow[t]{2}{*}{$\mathrm{Ni}$} & $\mathrm{R}$ & $1.70 \pm 0.13$ & $1.60 \pm 0.14$ & $0.73 \pm 0.16$ & $0.73 \pm 0.18$ & $0.76 \pm 0.20$ & $0.94 \pm 0.22$ & $1.9 \pm 0.3$ \\
\hline & M & $1.65 \pm 0.10$ & $1.52 \pm 0.14$ & $0.99 \pm 0.10$ & $0.64 \pm 0.20$ & $0.69 \pm 0.17$ & un. & un. \\
\hline \multirow[t]{2}{*}{$\chi^{2} /$ d.o.f. } & $\mathrm{R}$ & $726 / 525$ & $764 / 525$ & $814 / 525$ & $721 / 525$ & $653 / 525$ & $712 / 499$ & $1281 / 499$ \\
\hline & M & $782 / 525$ & $664 / 525$ & $707 / 525$ & $596 / 525$ & $532 / 525$ & $607 / 499$ & $923 / 499$ \\
\hline
\end{tabular}

